\title{
Neural Anatomy and Optical Microscopy (NAOMi) Simulation for evaluating calcium imaging methods
}

\author{
Adam S. Charles ${ }^{1 *}$, Alexander Song ${ }^{2 *}$, Jeff L. Gauthier ${ }^{1}$, Jonathan W. Pillow ${ }^{1,3}$, and David \\ W. Tank ${ }^{1,4,5}$ \\ ${ }^{1}$ Princeton Neuroscience Institute, Princeton University \\ ${ }^{2}$ Department of Physics, Princeton University \\ ${ }^{3}$ Department of Psychology, Princeton University \\ ${ }^{4}$ Bezos Center for Neural Circuit Dynamics, Princeton University \\ ${ }^{5}$ Department of Molecular Biology, Princeton University \\ *Equal contribution
}

\begin{abstract}
The past decade has seen a multitude of new in vivo functional imaging methodologies. However, the lack of ground-truth comparisons or evaluation metrics makes large-scale, systematic validation impossible. Here we provide a new framework for evaluating TPM methods via in silico Neural Anatomy and Optical Microscopy (NAOMi) simulation. Our computationally efficient model generates large anatomical volumes of mouse cortex, simulates neural activity, and incorporates optical propagation and scanning to create realistic calcium imaging datasets. We verify NAOMi simulations against in vivo two-photon recordings from mouse cortex. We leverage this access to in silico ground truth to perform direct comparisons between different segmentation algorithms and optical designs. We find modern segmentation algorithms extract strong neural time-courses comparable to estimation using oracle spatial information, but with an increase in the false positive rate. Comparison between optical setups demonstrate improved resilience to motion artifacts in sparsely labeled samples using Bessel beams, increased signal-to-noise ratio and cell-count using low numerical aperture Gaussian beams and nuclear GCaMP, and more uniform spatial sampling with temporal focusing versus multi-plane imaging. Overall, by leveraging the rich accumulated knowledge of neural anatomy and optical physics, we provide a powerful new tool to assess and develop important methods in neural imaging.
\end{abstract}

\section{Introduction}

The endeavor to understand neural systems has spurred rapid development of technology that can record brain activity at ever larger scales [1-3] and higher precision [4-6]. One such class of technology, functional optical microscopy, has empowered researchers to explore neural dynamics from synapse $[7,8]$ to large brain regions $[9,10]$. Specifically, two-photon microscopy (TPM) combined with in vivo calcium imaging [11-16], has enabled the simultaneous recording of unprecedented numbers of neurons (over 9000) at cellular resolution [17, 18]. 
Although TPM has found widespread use [19-21], many available experimental techniques and data processing algorithms lack appropriate, systematic assessment [22, 23]. This deficit can result in inaccurate interpretation of neural data [24]. A systematic comparison of techniques would allow researchers to make better informed decisions about equipment and data-processing.

For instance, while imaging deeper into scattering tissue with TPM can benefit from decreasing the excitation numerical aperture (NA) [15], it is unknown how this benefit interacts with other optical or experimental design choices, such as adaptive optics [25, 26] or dendritic imaging [27, 28]. Additionally, while many algorithms have been designed to extract the neural activity traces and spatial profiles from TPM data [29-43], few options exist to assess the fidelity of the inferred segmentation beyond comparisons to manually annotated data [24, 44, 45].

In both cases assessment suffers from a lack of ground truth data, the gold standard of which requires simultaneous intracellular electrophysiological and TPM recordings [46, 47]. Such experiments are both difficult to perform and limited to only a few neurons and imaging conditions. The small number of neurons from such experiments limits the assessment scope by biasing towards cells that are in focus, fire often, and fluoresce brightly. This problem is further exacerbated as assessing multiple imaging parameters requires recordings under each imaging condition, greatly increasing the cost of collecting such data.

Alternatively, subjective ground truth can be obtained from TPM recordings via manual annotation [48]. Human labels, however, do not provide access to the underlying neural spiking, are limited by the same signal-to-noise ratio (SNR) that limit demixing algorithms, and may also bias analysis against dim or sparsely firing neurons. These same issues also affect comparisons using simultaneous conventional TPM recordings to test novel imaging conditions $[49,50]$.

In place of collecting ground truth data, simulations can provide rich, controlled testing data. Such approaches have benefited other imaging modalities, such as fMRI [51]. Simulation-based approaches, however, often suffer from being either too simple or too complex. While simple simulations are computationally efficient, they often only create realizations of the model being tested rather than the actual underlying phenomenon [52, 53]. Complex simulations instead capture too much detail and are severely limited computationally, requiring high-performance computing to simulate more than small volumes with a handful of neurons [54, 55]. For these reasons, existing methods do not provide plausible and computationally efficient simulations useful for large-scale functional imaging.

To assess TPM methods with realistic and computationally efficient simulations, we present the Neural Anatomy and Optical Microscopy (NAOMi) simulator. Our framework leverages simple, but flexible, models of neural tissue to efficiently create large volumes with thousands of neurons on standard workstations (Fig. 1). Arbitrary patterns of spiking activity can be generated for this population, which our framework then transforms into realistic fluorescence traces separately for somas as well as processes. A light model approximates laser propagation and scattering throughout different locations of the simulated tissue. These components are combined in a simulated scanning procedure that incorporates important imaging effects, such as sample motion. We describe the simulation model, which has a publicly available software implementation ${ }^{1}$, and provide parameters for simulating two-photon GCaMP [46] recordings in layer 2/3 of mouse visual cortex. We use these simulated datasets to evaluate several automated calcium imaging demixing algorithms. Finally, we generate several more datasets to compare the performance of standard and specialized TPM experimental setups under a variety of sample conditions.

\footnotetext{
${ }^{1}$ Code will be made available post-review. Please contact A. Charles and A. Song for beta testing requests.
} 


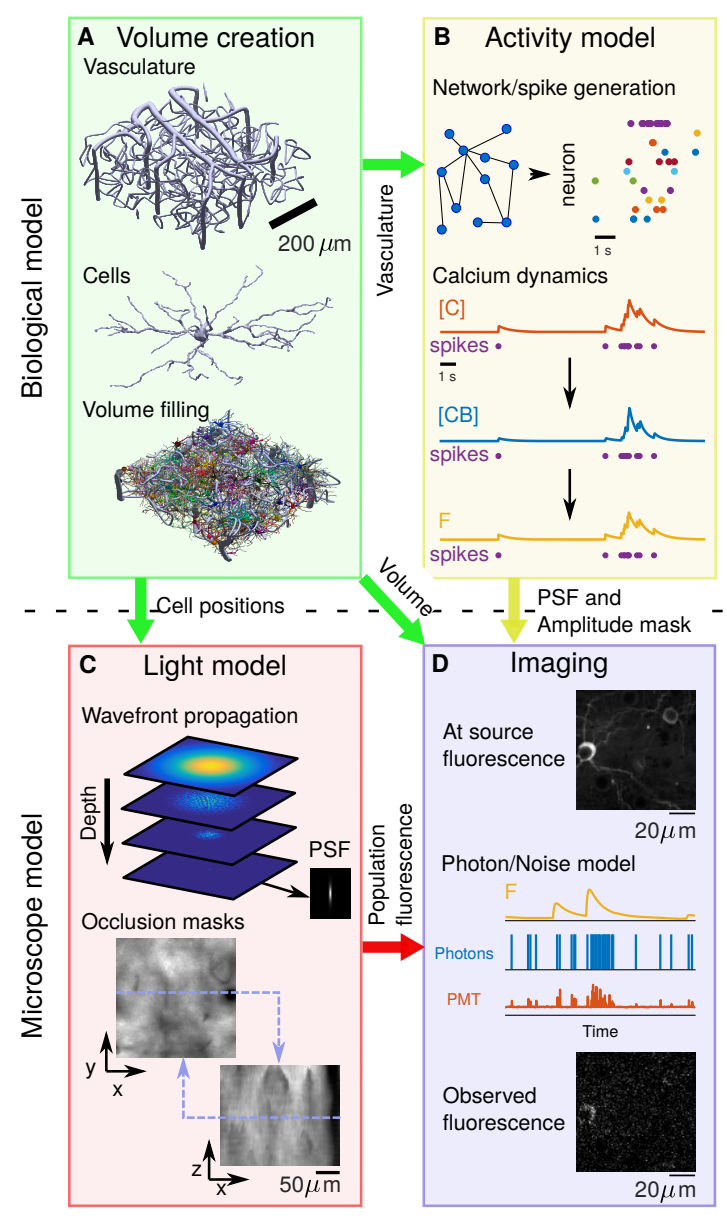

Figure 1: Block diagram of NAOMi simulator. A: Neural volume generation process. Vasculature is generated throughout the volume, followed by cell bodies and finally dendrites and axons are grown. B: Network activity generation. The spiking activity for each neuron is simulated and converted into calcium $([\mathrm{C}])$, bound calcium ([CB]), and fluorescence $(\mathrm{F})$ for a chosen indicator. C: Light propagation model. An optical wavefront corresponding to particular microscope optics is propagated through a simulated scattering volume, generating a scattered point-spread function and relative intensity masks. D: Scanning and image formation. The volume, modulated by the simulated activity, is scanned using the output of the light model with motion and noise sources from a model of the light collection, amplification, and digitization process.

\section{Results}

\subsection{Simulation design}

Generating realistic imaging data useful for honest assessment of a spectrum of techniques hinges on accurate, efficient simulations of anatomical volumes at the scale of optical imaging (Fig. 1A). Our anatomical simulation starts by constructing a scaffolding of vasculature with three parts: surface vessels, diving vessels, and capillaries [56] (Tab. 1, Sup. Fig. 1, 2, 3, See Methods). Next neurons are placed throughout the volume, first placing somas, and then growing dendrites and axons [57] 
from the cell bodies (Sup. Fig. 4, 5, See Methods). Statistical models of neurons and process paths ensure variation in cell shapes and were tuned using morphological data from electron microscopy (EM) data [58] and optical microscopy [59-61] (Tab. 1, See Methods).

The next step in simulating TPM data is to augment each generated neuron with realistic fluorescence activity (Fig. 1B). Spiking activity for each neuron is either pre-defined or is generated using models that output correlated, bursting population activity based on models of neural connectivity [62-66] (see Methods). Next, the known non-linear calcium decay process simulates the dynamic concentration of calcium ions [67,68]. This two compartment model describes separate dynamics for the cell bodies and the neurites, both driven by the same spike trains. As in related work, a protein-specific double exponential model modulates the calcium concentrations to create bound calcium concentrations with appropriate onset and offset time-constants [68, 69]. Finally, the bound calcium concentrations are converted to fluorescence values using the Hill-equation fit to fluorescence measurements [70, 71] (Tab. 1, Sup. Fig. 8, see Methods).

The next step in the simulation is to estimate the optical properties of the specified microscope configuration within the generated tissue (Fig. 1C). The scattering nature of brain tissue substantially affects light propagation through it, resulting in an abberated point-spread function (PSF) and decreased optical performance. We approximate these effects by performing wavefront propagation of a specified beam shape (i.e. Gaussian or Bessel beams [72, 73]) through a generated volume of refractive index shifts (Sup. Fig. 10, See Methods), generating simulated PSFs across the volume. The weight of the simulated PSF across different locations of the simulated volume forms an occlusion mask, representing inhomogeneity of optical performance across the sample. This occlusion mask is also modulated by an estimate of the absorption of emitted light through blood vessels and the neural volume as a function of position.

The final scanning module combines the outputs of the anatomical, light, and activity modules to produce images on a frame-by-frame basis (Fig. 1D). The fluorescence activity and occlusion mask modulate the anatomical volume, which is convolved with the simulated PSF to produce raw, noiseless, illumination images. Sub-pixel line-by-line offsets, representing brain motion, are applied prior to spatially resampling to the desired image resolution and applying the measurement noise model. Measurement noise is simulated by per-pixel Poisson sampling of photons counts at the photo-multiplier tube (PMT) and converting these counts into electrical measurements via PMT photon and electronics amplification distributions (Sup. Fig. 10, see Methods). This process includes bleed-through across pixels from the amplifier's temporal response kernel (Sup. Fig. 12, see Methods). This procedure is performed independently for each frame, and captures the complex, non-Gaussian noise profile inherent in TPM data.

\subsection{Comparison of simulated data to real data}

We next evaluate the overall simulation output against recordings from mouse V1 (Fig. 2). We generated a $500 \mu \mathrm{m} \times 500 \mu \mathrm{m} \times 100 \mu \mathrm{m}$ volume and scanned it with a 0.6-NA Gaussian point-spread function over 20,000 frames at $30 \mathrm{~Hz}$ sampling and $40 \mathrm{~mW}$ laser power, comparable to parameters in a recorded dataset obtained from mice expressing GCaMP6f being exposed to a set of visual stimuli (see Methods).

The simulated videos and recorded videos visually share many of the same features (Sup. Video 1), including bright, sparse transients of fluorescence across the whole image. The overall mean images (average of frames across time; Fig. 2A) both show distinct cell bodies along with muted processes that have their intensity modulated by scattering from blood vessels and other tissue 


\begin{tabular}{|c|c|c|c|c|c|}
\hline Parameter & Lit. Val. & Fit type & $\begin{array}{c}\text { NAOMi } \\
\text { Val. }\end{array}$ & Unit & Refs \\
\hline \multicolumn{6}{|l|}{ Anatomical parameters: } \\
\hline Neural density & $9.20 \mathrm{E}+04$ & Direct & - & $\mathrm{mm}^{-3}$ & {$[58]$} \\
\hline Fraction vasculature & $0.01-0.04$ & Indirect & 0.032 & - & {$[58,74,75]$} \\
\hline Fraction cell bodies & 0.12 & Indirect & 0.135 & - & {$[58]$} \\
\hline Fraction neuropil & 0.84 & Indirect & 0.833 & - & [58] \\
\hline Fraction dendrites* & 0.294 & Indirect & 0.223 & - & {$[58]$} \\
\hline Fraction other (fluorescing)* & 0.401 & Indirect & 0.33 & - & {$[58]$} \\
\hline Fraction other (not fluorescing)* & 0.293 & Indirect & 0.28 & - & {$[58,76]$} \\
\hline Vessel radius (capillary) & $2.00 \mathrm{E}+00$ & Direct & - & $\mu \mathrm{m}$ & {$[56]$} \\
\hline Vessel radius (penetrating) & $10(9,11)$ & Direct & - & $\mu \mathrm{m}$ & {$[56]$} \\
\hline Vascular density & $1-3$ & Indirect & 2 & $\%$ & {$[56,58,74,75]$} \\
\hline Penetrating vessel density & $30^{\dagger}$ & Direct & - & $\mathrm{mm}^{-2}$ & {$[56]$} \\
\hline Somatic volume* & $1.80 \mathrm{E}+03^{\dagger}$ & Indirect & $1.80 \mathrm{E}+03$ & $\mu \mathrm{m}^{3}$ & {$[77]$} \\
\hline Nuclear volume* & $800^{\dagger}$ & Indirect & 800 & $\mu \mathrm{m}^{3}$ & {$[77]$} \\
\hline Cytoplasm volume* & $1000^{\dagger}$ & Indirect & 1000 & $\mu \mathrm{m}^{3}$ & {$[77]$} \\
\hline Basal dendrite diameter & 0.7 & Direct & - & $\mu \mathrm{m}$ & {$[78]$} \\
\hline Basal dendrite length & $100-160^{\dagger}$ & Indirect & 105 & $\mu \mathrm{m}$ & {$[59-61]$} \\
\hline Apical dendrite diameter & $1-2$ & Direct & - & $\mu \mathrm{m}$ & [79] \\
\hline Axonal diameter & 0.3 & Direct & - & $\mu \mathrm{m}$ & {$[58]$} \\
\hline \multicolumn{6}{|l|}{ Fluorescence parameters: } \\
\hline GCaMP6f binding affinity $K_{d}$ & 290 & Direct & - & nMol & {$[70]$} \\
\hline Baseline $\mathrm{Ca}^{2+}$ concentration & 50.00 & Direct & - & nMol & {$[68]$} \\
\hline $\mathrm{Ca}^{2+}$ binding ratio $k_{s}$ & 100,110 & Direct & 110 & $\mathrm{AU}$ & {$[67,80-82]$} \\
\hline $\mathrm{Ca}^{2+}$ diffusion constant $\gamma$ & 1800 & Data fit & 292.3 & $\mathrm{~s}^{-1}$ & {$[67,80,83]$} \\
\hline $\mathrm{Ca}^{2+}$ axon diffusion constant $\gamma$ & 2800 & Direct & - & $\mathrm{s}^{-1}$ & {$[67,80,81]$} \\
\hline GCaMP6f Hill eqn. exponent $n_{h}$ & 2.7 & Direct & - & $\mathrm{AU}$ & {$[70]$} \\
\hline GCaMP6f Hill eqn. amplitude & 25.2 & Direct & - & $\mathrm{F}$ & {$[70]$} \\
\hline Indicator concentration & $10-200$ & Direct & 10 & $\mu \mathrm{M}$ & {$[68,84,85]$} \\
\hline
\end{tabular}

Table 1: Parameters used for in-silico simulation of neural activity in layer II/III of mouse primary visual area V1. Values for each parameter were either directly found in the literature or estimated from published data (entries with a $\dagger$ ). The third column indicates whether these parameters were set directly in NAOMi, or were fit indirectly by setting other simulation parameters. In the latter cases, the measured values from a simulated NAOMi volume are shown for comparison, indicating that the simulated anatomy matches measured anatomical statistics.

elements. Histograms of video pixel values (Fig. 2A,B) feature heavy right tails corresponding to neural activity and also peaks at zero corresponding to zero-photon pixels.

The neural activity distribution for individual pixels is explored by comparing the relative strength of firing activity across the field of view (FOV). The distribution of maximum activity 
(maximum $\Delta \mathrm{F} / \mathrm{F}$ over 20,000 frames) for all pixels (Fig. 2C) for each of the two videos is peaked at 2 with a slight heavy right tail, which corresponds with neurons that fired large transients within the videos. Other statistics, such as the distribution of values in the mean image, the standard deviations over all pixels, and measures of activity such as the ratios of maximum to median fluorescence values also match well (Sup. Fig. 14A-C).

The global frequency content of the two videos is estimated with the $2 \mathrm{D}$ discrete Fourier transforms of the mean images (Fig. 2D). The Fourier transforms of both videos depict very similar features, such as increased frequency content along the fast- and slow-scanning axes resulting from the sequential pixel bleed-through and residual line-by-line motion artifacts. Additionally, the frequency fall-off (Fig. 2E) for both the real and simulated data display the same decay. Finally, observing the effective dimensionality of both videos via Principal Component Analysis (PCA; Fig. 2F) finds both qualitative similarities between the spatial principal components (PCs) and quantitative similarities between the distribution of variance explained for the leading PCs on small patches of videos.

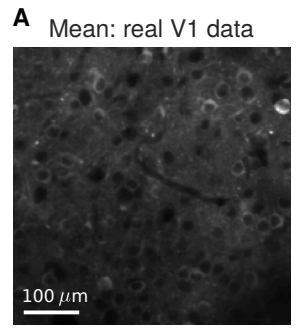

D DFT: real V1 data

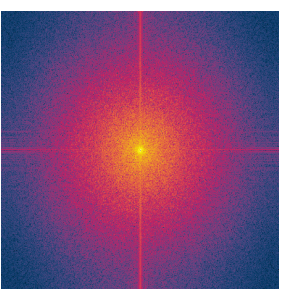

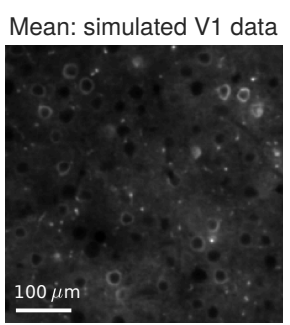
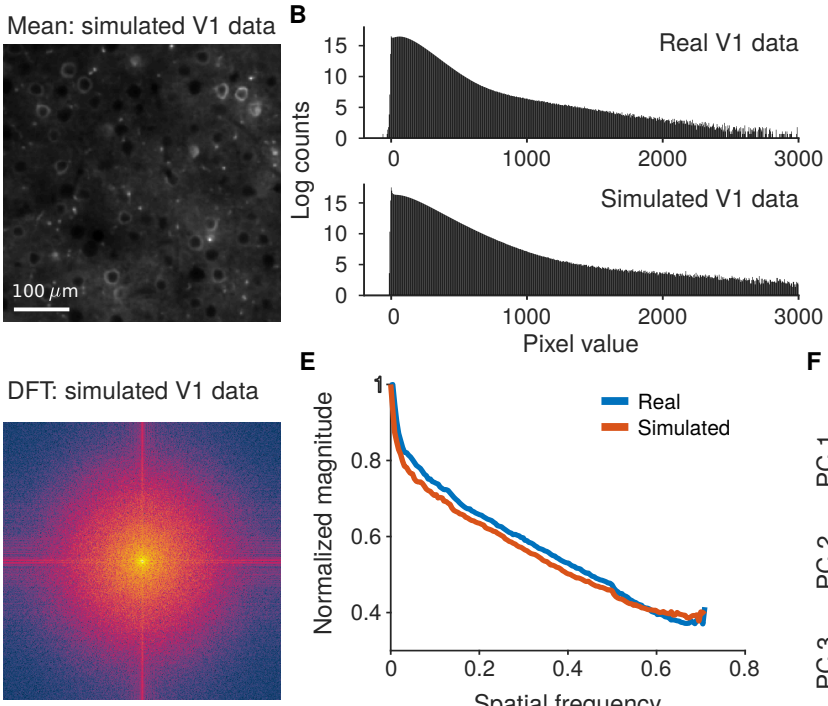

$\mathrm{E}$

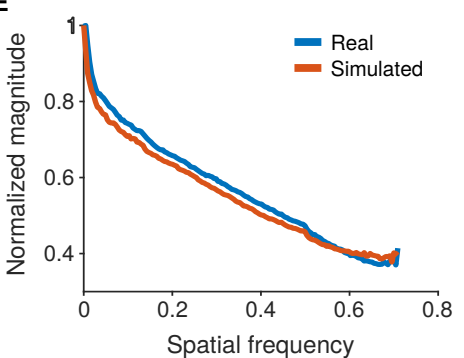

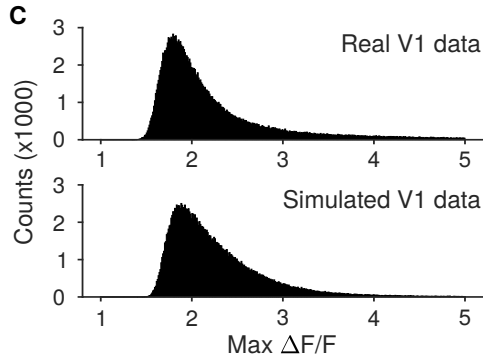

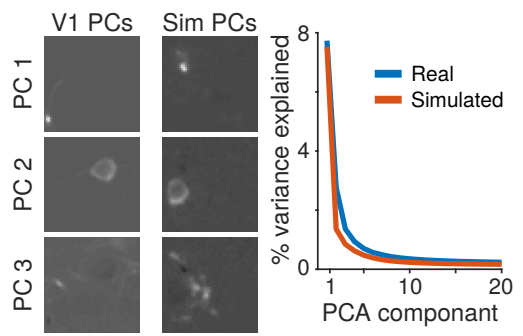

Figure 2: Comparison of simulated data to recordings of mouse V1 L2/3 using GCaMP6f. A: The mean image for mouse V1 recordings and simulated data. B: Pixel value distributions across the full videos display bimodal peaks and a right log-linear tail. $\mathrm{C}$ : Distribution of the maximum $\Delta \mathrm{F} / \mathrm{F}$ values across all pixels in the FOV match between the simulated and real V1 data. D: The spatial frequency content in the mean simulated image captures the qualities of the real data. Both the spread of frequencies and the tendency for high-frequency components in the fast- and slow- scan directions that result from line-by-line motion and pixel bleed-through are captured. E: The overall contributions at different spatial frequencies to the mean activity matches between the recording and simulation. F: Principal component decompositions for both the real and simulated data exhibit similar decays in the variance explained per component. The resulting spatial principal components are qualitatively similar. 


\subsection{Evaluation of automated segmentation}

We start evaluating TPM techniques using NAOMi by analyzing the performance of automated demixing algorithms, leveraging the ground truth information available. We applied three common algorithms - PCA/ICA [29], constrained non-negative matrix factorization (CNMF) [38], and Suite2p [18] — to 20,000 frames simulated from a $500 \mu \mathrm{m}$ x $500 \mu \mathrm{m}$ x $100 \mu \mathrm{m}$ volume with $1 \mu \mathrm{m}$ sampling at $30 \mathrm{~Hz}$ scanning using a 0.6-NA Gaussian excitation numerical aperture (NA) at $40 \mathrm{~mW}$ average power. The ground truth consisted of the spatial profiles of each individual neuron and component within the volume and their individual fluorescence traces.

Each algorithm returned a set of demixed time traces and corresponding spatial profiles (Tab. 68, Sup. Fig. 16- 19). Overall CNMF, Suite2p and PCA/ICA isolated 1091, 661, and 265 components, respectively, out of a total of 8,117 possible fluorescing components. Comparisons to the ground truth traces, based on a combined Pearson's correlation cut-off of 0.1 on the time-traces and a $50 \%$ pixel overlap, revealed which components represented actual cells in the volume (Tab. 6). We considered a pairing to be a "strong pairing" if the correlation exceeded 0.5 (Fig. 3A,B). These correlation values account for all aspects of how well the estimated traces match the true timecourses, including missed transients and false transients from other components (e.g., neuropil; Fig. 3C).

Of the paired profiles, some were doubled, i.e. multiple algorithmically discovered profiles matched to different portions of the same simulated cell (Sup. Fig. 23, Tab. 6). Accounting for doubling, CNMF, Suite2p, and PCA/ICA found 303, 292, and 137 unique cells at the $>0.5$ correlation level. Interestingly, while CNMF finds the most distinct components (i.e., before accounting for cells found with multiplicity), it only finds approximately the same number of unique cells as Suite2p, and both have a lower rate of found true cells than PCA/ICA (Fig. 3D). Furthermore, comparisons of individual cells found (Tab. 7, 8) show that different methods find non-overlapping sets of cells (Sup. Fig. 21). For example, CNMF and Suite2p only agree on 273 of the $\approx 300$ cells (Fig. 3A, Tab. 7).

While these figures may seem small compared to the 8,117 total sources, not all fluorescence sources are visible above the noise level. To explore this effect with NAOMi, we computed auxiliary time-traces from the raw, noisy video using the "ideal" ground-truth spatial profiles to obtain the profile-aware least-squares (PALS) time trace estimates (see Methods). Due to the video signal-tonoise ratio (SNR), these estimates yielded only 415 timecourses accurately matched at the $>0.5$ correlation level (Fig. 3D, Tab. 8) indicating that the gap induced by simultaneous estimation of spatial profiles is not overly large. In fact, the inherent denoising in some algorithms allows some cells' time courses to be estimated with even higher fidelity than the traces derived from the ideal spatial profiles (e.g. CNMF identified 8 cells at the $r>0.5$ level that the ideal profiles produced lower correlation values for; Tab. 8).

One challenge in interpreting the results of automated demixing is that, sans ground-truth, it is difficult to determine if a source is a true cell or an artifact. Instead, sorting components based on metrics such as overall fluorescence levels can be used. Varying one such criterion - a threshold on the maximum fluorescence - to classify true and artifact sources results in receiver-operator characteristic (ROC) curves that compare the number of strongly paired components kept (true positives), to the number of weakly paired or unpaired components kept (false positives). These curves show that while PCA/ICA obtained the fewest components overall, CNMF and Suite2p found bright artifacts at much higher rates (Fig. 3E, Sup. Fig. 24).

One benefit of the NAOMi simulator is that we can easily explore how optical parameters 
effect algorithmic performance. We replicated the above analysis with a $2 \mathrm{x}$ increase in laser power $(80 \mathrm{~mW})$, keeping the volume and neural activity constant. Ideally this power boost would illuminate additional cells, as weaker and more sparsely firing cells would be more distinguishable. We found that all algorithms returned more unique components at the $r>0.5$ level, with 424 for CNMF, 358 with Suite2p and 264 for PCA/ICA: a 39.93\%, 22.6\%, and 92.7\% improvement (Sup. Tab. 9), respectively. Interestingly, a similar increase in components that did not pair well to real cells meant that there was negligible improvement in rate of found cells, and some ROC curves reduced in area, indicating that fluorescence magnitudes became less sufficient to differentiate true cells from artifacts (Sup. Fig. 24.

We note that in addition to laser power, other factors such as the sampling resolution, numerical aperture and neuropil strength also influence the ability to detect neural activity. NAOMi enables exploration of all these aspects. For example we find 1) a sharp cut-off in the ability to accurately detect components when sampling at intervals larger than $3 \mu \mathrm{m}$ (Sup. Fig. 25) 2) an improvement in the ability to detect smaller burst sizes in the $\Delta \mathrm{F} / \mathrm{F}$ values with neuropil correction as in Suite2P [18] (Sup. Fig. 26), and 3) a steady decay in signal strength per component as a function of NA, reaching a critical reduction of signal at $\mathrm{NA} \approx 4$ (Sup. Fig. 27 ).

\subsection{Evaluation of TPM optical configurations}

The ability to modify optical parameters and sample expression patterns allowed for direct assessment of the trade-offs between microscope configurations across sample conditions. We applied this new mode of assessment to perform three head-to-head comparisons: 1) imaging of sparsely labeled tissue using Bessel [8] vs. high-NA Gaussian beams (Fig. 4A-C), 2) imaging of nuclear labeled tissue using high-NA vs. low-NA (axially extended) Gaussian beams (Fig. 4D-F), and 3) volumetric imaging of densely labeled tissue using multiplane Gaussian [87] vs. temporally-focused beams [88] (Fig. 4G-I).

In our first comparison we simulated TPM recordings of a sparsely labeled ( $10 \%$ neurons expressing GCaMP6f) $500 \mu \mathrm{m}$ x $500 \mu \mathrm{m}$ FOV of mouse cortex using both conventional high-resolution TPM with a Gaussian PSF (0.6 NA) and extended depth-of-field TPM using a Bessel beam (0.4 NA, $60 \mu \mathrm{m}$ long) [8]. As shown previously, Bessel beam imaging resulted in excitation throughout the whole volume, resulting in more uniform excitation of neurons (Fig. 4C) and more neurons recorded with high signal fidelity. Another consequence of the uniform excitation observed using Bessel beams is an increased robustness to axial motion. To test this, we clustered the extracted time-traces from Bessel and Gaussian imaging into three groups (Fig. 4B). The sorted correlation matrices showed a strong motion-induced artifact only for Gaussian imaging, suggesting a reduced influence of motion artifacts on time-trace estimation using Bessel beams.

Our second comparison tested the performance of nuclear labeled TPM, which has been extensively used for larval zebrafish and $C$. elegans TPM [89, 90] but not in mouse brain imaging, in a $500 \mu \mathrm{m} \times 500 \mu \mathrm{m}$ FOV of mouse cortex. We imaged this simulated tissue using Gaussian beams of two excitation NAs: 0.2 and 0.6 (Fig. 4D). Surprisingly, simulations of signal levels across a variety imaging NA values showed constant overall integrated value (i.e., signal level; Sup. Fig. 27), suggesting little loss of power efficiency by reducing the excitation NA. Similar to the extended depth-of-field Bessel beam, the 0.2 NA excitation resulted in sampling more neurons from the volume and improved overall imaging performance (Fig. 4E,F). As the imaging volume is mostly non-fluorescing with nuclear-labeling, distinguishing individual cells is straightforward even with a extended depth-of-field. Because nuclei are several times larger than the lateral resolution of the 

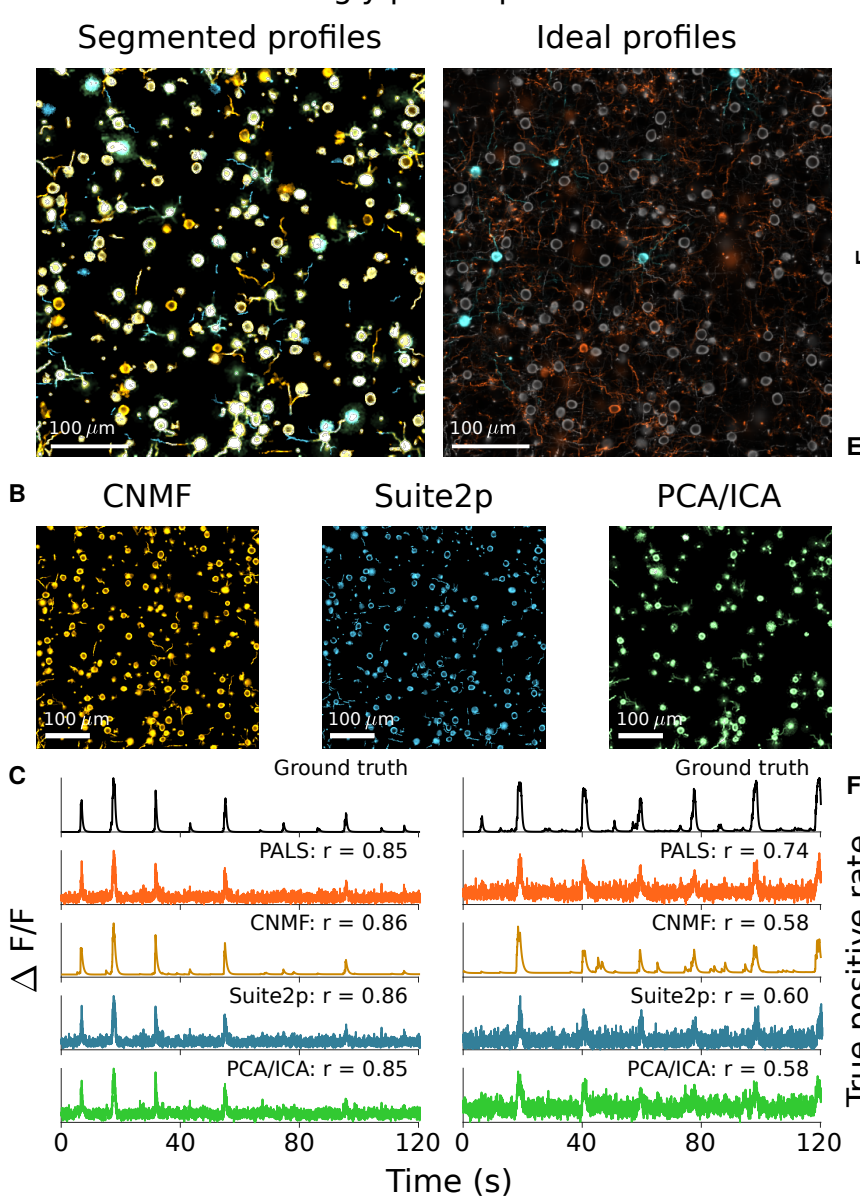

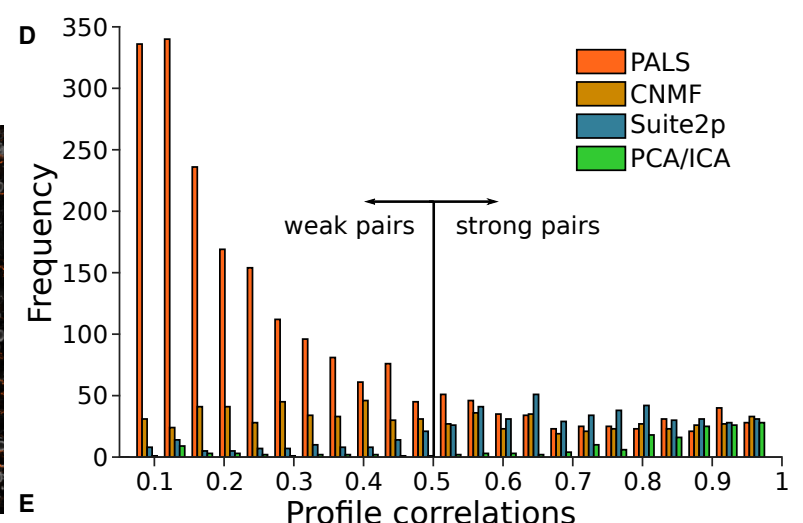

Strongly paired Weakly paired Unpaired
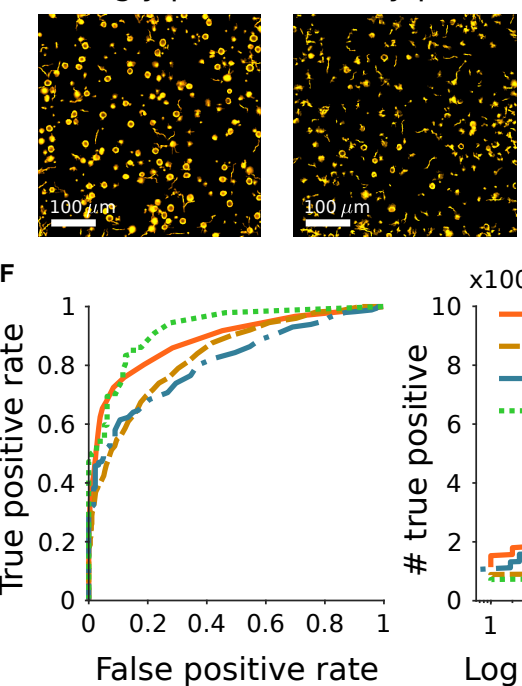
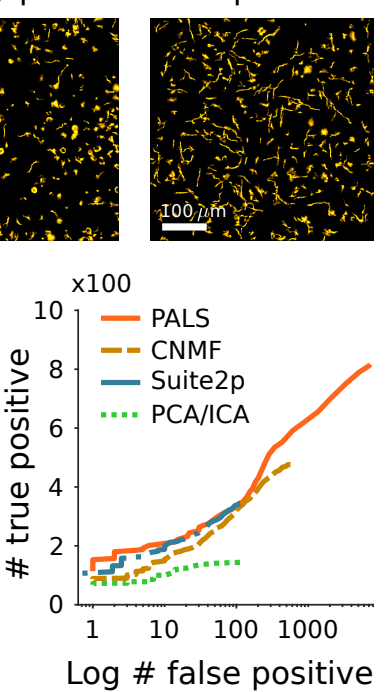

Figure 3: Comparison of popular calcium imaging segmentation algorithms using synthetic data generated using NAOMI. A: Spatial profiles from CNMF (red), Suite2p (blue), and PCA/ICA (green) overlaid with ideal profiles (yellow) calculated from the simulation volume. B: Strongly paired $(\mathrm{r}>=0.5)$ spatial profiles from each algorithm displayed separately. C: Example timecourses estimated by each of the segmentation algorithms as compared to the ideal profile assisted leastsquares (PALS) estimated timecourse and the ground truth timecourse. D: Histogram of correlation values of estimated timecourses to the ground truth timecourses for each match spatial profile. E: Spatial profiles from CNMF separated into strongly paired $(\mathrm{r}>=0.5)$, weakly paired $(\mathrm{r}<=0.5)$ or unpaired. F: ROC curves for strongly paired $(\mathrm{r}>=0.5)$ spatial profiles sorted by their peak fluorescence and profile weight.

0.2 NA excitation beam, there are fewer advantages to switching to a Bessel beam setup and low NA imaging is more power efficient.

Last, we compared multiplane TPM [87] to scanned temporal focused (s-TeFo) TPM [88] in a $500 \mu \mathrm{m} \times 500 \mu \mathrm{m} \times 200 \mu \mathrm{m}$ volume of the mouse cortex layers 2-4 (Fig. 4G). For multiplane Gaussian TPM, we scanned four planes, separated by $50 \mu \mathrm{m}$ at $1 \mu \mathrm{m}$ lateral sampling, at $10 \mathrm{~Hz}$ and for s-TeFo TPM, we scanned 16 planes, separated by $10 \mu \mathrm{m}$ at $4 \mu \mathrm{m}$ lateral sampling, at $10 \mathrm{~Hz}$. Given a similar amount of excitation within the volume, both techniques performed comparably in 
extracting fluorescence traces with high fidelity. The axial distribution of highly correlated $(\mathrm{r}>$ 0.5) cells in the multiplane TPM is multimodal, set at the focal positions the four planes, while the $\mathrm{s}-\mathrm{TeFo}$ distribution is much more uniform (Fig. $4 \mathrm{H}$ ), as is reflected in the profile weight histograms (Fig. 4I). These simulations suggest that while s-TeFo as a technique may not drastically increase the number of recorded neurons, the more consistent spatio-temporal sampling of neurons may decrease sampling bias and provide robustness to motion-induced artifacts and signal crosstalk.

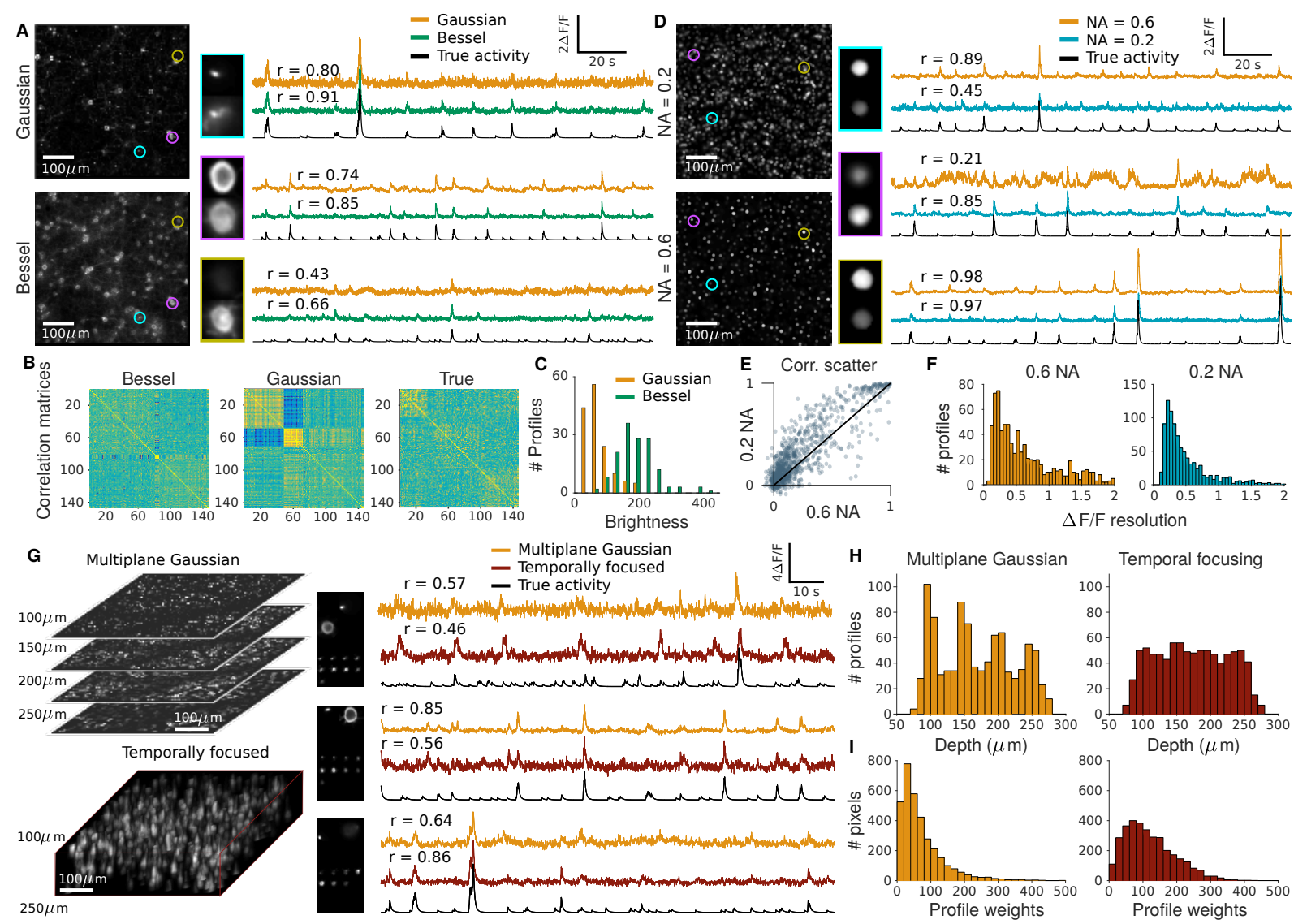

Figure 4: Comparison of specialized imaging modalities to standard high NA Gaussian TPM for a sparsely labeled volume (A-D), nuclear labeled volume (E-H), and volumetric imaging (I-L). A: Mean image (left) and example time traces (right) of a sparsely labeled volume with Bessel beam (top) and high NA Gaussian (bottom) illumination. B: Correlation matrices of extracted time traces for each method sorted by clustering into 3 groups using k-means. C: Histograms of spatial profile weights of cells in the volume using Gaussian and Bessel PSFs. D: Mean image (left) and example time traces (right) of a nuclear labeled volume with low NA Gaussian (top) and high NA Gaussian (bottom) illumination. E: Scatterplot of correlation values for low and high NA extracted timecourses against the true timecourse. F: Estimated $\Delta F / F$ resolution of cells based on their spatial profile and the mean image. G: Mean image (left) and example time traces (right) of volumetric TPM with 4 high NA Gaussian planes (top) and 16 temporally focused planes (bottom) illumination. H: Histogram of axial positions of highly correlated $(\mathrm{r}>0.5)$ cells using a scanned high NA Gaussian or temporally focused illumination. I: Histograms of profile weights using high NA Gaussian or temporally focused illumination. 


\section{Discussion}

We present here the NAOMi simulation framework that generates detailed TPM data. This framework yields new insights into TPM technology and enables the testing and validation of existing and novel TPM methods, allowing for assessment and methodological optimization not currently possible. Toward this end, we have developed the ability to generate realistic synthetic neural volumes, transient calcium activity, and two-photon calcium imaging videos. We further increase the simulation efficiency by simplifying statistical models of the processes involved, reducing the computational burden and making this tool more broadly applicable.

We have demonstrated two important use cases of NAOMi: assessment of calcium imaging demixing methods and comparing optical configurations across imaging conditions. In both cases, useful large-scale ground truth is difficult to obtain experimentally. NAOMi leverages the accumulated knowledge of neuroanatomy, optical physics, and neuroscience to bypass these difficulties through simulation.

To assess demixing algorithms, we generated a calcium imaging dataset typical of TPM in mouse neocortex and analyzed the performance of several popular methods: CNMF [52], Suite2p [34], and PCA/ICA [29]. The available input cell shapes and timecourses enabled direct comparisons of the algorithm decompositions to ground truth, which revealed that methods with the highest number of found neurons also had much higher instances of artifactual components. This indicates the potential for many false positives in automated demixing (Sup. Fig. 20, Tab. 6). Using NAOMi data as a testing ground, more advanced detection metrics can be developed, to find better ways to filter out true cells from the full set of returned components.

Using NAOMi, we explored particular combinations of optics and samples in silico and illustrated the advantages three specialized techniques could have while imaging particular sample conditions. We rapidly assessed whether or not a particular technique is appropriate for the sample and optimized imaging parameters to maximize the quantity and quality of the data we collect. Additionally, we explored the interaction of different components of in vivo calcium imaging and their effects on performance. For instance, we quantified the effects that axial brain motion can have on estimated cell-cell correlation as a function of both sample type and optical configuration.

Despite these results, there are several aspects of the simulation that were simplified for speed or simplicity. While we provide a rudimentary spike-train simulation to drive the fluorescence models, we note that more realistic firing patterns for neurons will help improve overall realism. A number of complex packages simulate such activity for neural populations (e.g. [91]). In these cases, rather than replicate these methods, we encourage their use and integration into the NAOMi framework.

In the development of NAOMi we aimed to create a tool accessible to the community at large that is easily expandable in its scope and abilities. To this end, our software was designed to be modular so that as better anatomical models, optical descriptions of tissue, and TPM statistics become available, they can be easily incorporated into the existing framework. Additionally, single modules can be modified in order to simulate different setups. For example, changing the anatomical structure (e.g., blood-vessel size and cell body statistics) can allow for bench-marking imaging techniques in rats, rather than mice. These changes and extensions will allow NAOMi to be a useful tool for a wide variety of applications for experimentalists and methods developers.

Work in other fields has shown the great utility of developing strong simulation-based models of experimental data (see [92] and references therein). NAOMi is a tool that fills part of this gap for neuroscience data. As neuroscience continues maturing, better models of data must be developed, 
especially for data that is as diverse and complex as two-photon calcium imaging. This and other work will allow researchers to not only more quantitatively judge the quality of their data, but also make better predictions on the data they will need for their experiments.

\section{Materials and Methods}

Our TPM simulator is designed to permit testing of many different aspects of the calcium imaging process. To achieve this flexibility, our simulator is divided into five distinct modules, each focused on a portion of either the tissue or scanning simulation (Fig. 1). The five modules are: 1) the neuron module responsible for generating single neurons, 2) the volume module responsible for assembling the neurons into a tissue volume that includes neuropil and vasculature, 3) the activity module that generates the temporal calcium traces for each neuron and the neuropil, 4) the optics module that simulates the point-spread function and occlusion due to the optical mask, and 5) the imaging module that simulates the TPM noise model and object motion.

\subsection{Neuron model}

The first module creates simple, yet plausible models of neurons that can be placed throughout a volume and scanned in simulation. We model the neural shape via a probability distribution over smooth deformation of a sphere, followed by a nonlinearity. This model allows for fast sampling of unique neurons, meaning that each simulated volume will contain a completely new set of neurons. Additionally, we provide for each neuron a nucleus modeled as a shrunken and smoothed version of the soma shape. This model captures the relationship observed in detailed field emission scanning electron microscopes (FESEM) [93]. Finally, we simulate for each neuron a number of dendrites, one of which is created thicker and at a downward orientation, as to model the apical dendrites.

The model of the smoothly deformed cell body is an isotropic Gaussian process [94] defined over a sphere. To sample from this distribution and create the cell body, we sample uniformly over a sphere [95], sample i.i.d. a Gaussian random variable for each point, and smooth the points according to the process covariance. Denoting the sample points $\boldsymbol{p}_{i} \in \mathbb{R}^{3}$, the height (distance from center of the sphere) can be sampled from

$$
r_{i} \sim \mathcal{N}(\mathbf{0}, \mathbb{K}), \quad \boldsymbol{K}_{i, j}=e^{-d\left(\boldsymbol{p}_{i}, \boldsymbol{p}_{j}\right) / l},
$$

where $l$ is the length-scale that controls the smoothness of the cell body, and $d(\cdot, \cdot)$ is the geodesic distance between any two points. For the unit sphere (radius one), this distance is the arc length along the great circle connecting the two points

$$
d\left(\boldsymbol{p}_{i}, \boldsymbol{p}_{j}\right)=2 \sin ^{-1}\left(\frac{\left\|\boldsymbol{p}_{i}-\boldsymbol{p}_{j}\right\|_{2}}{2}\right) .
$$

When unconstrained, the radial height of this function can, at times, exceed the maximum and minimum realistic deformations $r_{\max }=\max _{i}\left|r_{i}\right|$ and $r_{\min }=\min _{i}\left|r_{i}\right|$. We thus rescale the radii values as

$$
r_{i}=\left(r_{\max }-r_{\min }\right) \frac{\left|r_{i}\right|-\min _{i} r_{i}}{\max _{i} r_{i}-\min _{i} r_{i}}+r_{\min }
$$


The resulting points $r_{i} \boldsymbol{p}_{i}$ form the points for a mesh that define the interior of the cell body. To account for the non-spherical shape found in pyramidal neurons, we can modify the radii values by making the base radius at each point dependent on a function of its location on the sphere. Specifically, we use the equation for a tear-drop that is defined parametrically by the azimuth and elevation angles $\phi$, and $\theta$ as

$$
\left[\cos (\phi) \sin (\theta) \sin ^{m}\left(\frac{\theta}{2}\right), \sin (\phi) \sin (\theta) \sin ^{m}\left(\frac{\theta}{2}\right), \cos (\theta)\right] .
$$

The final step in creating the cell body is to create the nucleus, which is accomplished by shrinking and smoothing the cell wall shape as defined by $r_{i} \boldsymbol{p}_{i}$ as

$$
r_{i}^{*}=\left(r_{50 \%}\right) \frac{\left|r_{i}\right|-\min _{i} r_{i}}{r_{5 \%}+r_{i}}+r_{\min }-(\Delta r)_{\min }
$$

Dendrites are added to each neuron via a stochastic growing process [57]. The process generates start and end points for each dendrite, and iteratively grows the dendrite through the volume while avoiding any obstacles (i.e. other cell's somas, dendrites, or blood vessels). Apical and basal dendrite endpoints are separately set within the volume and the grown dendrites are dilated to widths consistent with measured anatomy (Tab. 1, Sup. Fig. 7).

\subsection{Volume generation}

To create the tissue volume, we initialize an empty volume and begin by placing blood vessels throughout the volume. For computational feasibility, the volume is modeled as a 3-D grid of points with sub-micron sampling (we typically use $0.5 \mu \mathrm{m}$ distances). The blood vessels are grown in three parts: surface vasculature, diving arterioles, and capillaries. Surface vasculature is grown by connecting nodes randomly placed upon the surface of the volume. The connected paths are smoothly varied and dilated. Diving arterioles are set at endpoints of surface vasculature and connected to the bottom of the simulated volume. Capillaries are connected to the diving arterioles and pseudo-randomly placed within the volume in a space-filling fashion. Vessel diameters, concentration, branching frequency, and orientation were compared and fit to two-photon microscopy data of mouse vasculature (Schaffer-Nishimura lab, unpublished data).

Once initialized, the volume is then filled with the neuron somas. We sequentially place the neurons randomly throughout the empty space in the volume, with a minimum distance that allows cell bodies, but not nuclei, to overlap. The random placement can be modified to encourage neurons to be more spread out, or more cluttered. When an overlap occurs, the overlapping region is given to the latest cell to be placed. This allows our volume to contain touching cell bodies. Once all the cell somas are placed, dendrites are grown for each neuron sequentially, such as to avoid location conflicts with other cells. Apical dendrites are grown in the same fashion, only thicker, axially oriented, and having fewer transversal deviations. Separate apical dendrites corresponding to neurons in deeper cell layers are grown in a similar fashion from the bottom of the volume to the top.

As a final step, axons fill up the remaining empty space, up to the typical 0.7 fill fraction of layer $2 / 3$ in mouse V1. The same dendrite growing algorithm [57] is used to create millions of short axon segments throughout the entire volume. To obtain the global correlated background components, 
we overlay 3D Gaussian processes that indicate the strength of each component throughout the volume with the set of axon locations. Specifically the value of the $k^{\text {th }}$ background component

$$
\boldsymbol{B}_{k}=\left|\boldsymbol{G}_{k}\right| \odot \boldsymbol{H}
$$

where $\odot$ represents element-wise multiplication between the 3 -tensors $\boldsymbol{H}$, which contains a one where a neuropil process is present and zero otherwise, and the absolute value of $\boldsymbol{G}_{k}$, a GP with mean zero and Gaussian radial-basis function covariance kernel where the covariance between any two points at locations $\boldsymbol{p}_{i}$ and $\boldsymbol{p}_{j}$ is

$$
\boldsymbol{K}_{i, j}=\rho e^{-\left\|\boldsymbol{p}_{i}-\boldsymbol{p}_{j}\right\|_{2}^{2} / l_{b g}^{2}}
$$

\subsection{Time-trace generation}

To simulate temporal activity, we provide a number of options to generate time-traces for each neuron. We provide both statistical models that generate stereotypical activity as well as more detailed $\left[\mathrm{Ca}^{2+}\right]$ dynamics model. The statistical model provides a simple way to input basic behaviors of various fluorescent proteins (i.e. rise-time and decay). The $\left[\mathrm{Ca}^{2+}\right]$ dynamics model simulates the molecular kinetics over time, and provides a way to test the time-trace assumptions made in calcium imaging analysis algorithms.

\subsubsection{Spike-time generation}

We provide two methods to generate spike trains to drive the fluorescence activity simulation. The first method creates independent activity for each neuron, including bursting behavior. The second model simulates a Hawkes process which accounts both for self-excitation, driving busting behavior, as well as inter-neuron spiking correlations [96].

To generate independent spike trains, we model each neuron as a bursting neuron, where bursts occur at independent, exponential intervals

$$
P\left(\Delta t_{\text {burst }}\right)=\lambda_{\text {burst }} e^{-\left(\Delta t_{\text {burst }}\right) / \lambda_{\text {burst }}}
$$

for $\Delta t_{\text {burst }}>0$. The rate of bursting $\lambda_{\text {burst }}$ is chosen differently for each neuron. The rates can be given to the simulator, or the simulator can automatically draw burst rates from a Gamma distribution with a provided mean rate and parameter $\alpha=1$. For each burst, the number of spikes are chosen as

$$
N_{\text {burst }}=1+\operatorname{Poisson}\left(\lambda_{N}\right)
$$

where the parameter $\lambda_{N}$ controls the length of the bursting. The inter-spike times between spikes in a burst were modeled as uniformly random between $5 \mathrm{~ms}$ and $7 \mathrm{~ms}$. Alternative distributions of spiking activities can easily be implemented by passing different $\lambda_{\text {burst }}$, $\alpha$, or $\lambda_{N}$ values to the simulator, or by direct modification to the code to implement different distributions that better reflect activity in other cortical areas.

For the Hawkes model simulation, we first generate a connectivity matrix that encodes how each neuron's firing excites other neurons. We model this connectivity with a Watts-Strogatz smallworld network model [62]. To correlate the processes to the network activity, we allow for all neurons to influence the background processes, while not allowing many return connections. To stabilize 
the point-process, we normalize the resulting connectivity matrix to have maximum eigenvalue magnitude of 0.98. We then run the Hawkes process using Lewis' method [97], with an exponential distribution over the neuron's base firing rates and a higher base firing rate for the background components. Finally, we bin the resulting continuous-time spike events into $1 \mathrm{~ms}$ bins to create the discretized spikes that are then fed into the calcium dynamics simulation.

\subsubsection{AR- $p$ dynamics}

For each cell, we generate a baseline fluorescence, $\beta_{i}=|1+z|$ where $z \sim \mathcal{N}\left(0, \sigma^{2}\right)$ is a Gaussian random variable. The variance $\sigma^{2}$ controls the distribution of baselines, and we set a default value to $\sigma^{2}=0.04$. The next step is to simulate the spike or "event" times for each neuron. As most neurons are sparsely active, we draw the firing rate of each neuron as $\lambda_{i} \sim \operatorname{Gamma}(a, \theta)$. The parameter $\theta$ gives the average inter-spike distance in time and should be set according to the temporal sampling rate set in the simulation. The parameter $a$ is the shape parameter and modulates the distribution of the firing rates. We find that $a=1$ (where the Gamma distribution collapses to an exponential distribution) yields realistic distributions of neuron activity levels. The actual event times are then sampled for each neuron according to a Poisson process with rate $\lambda_{i}$. To model the different calcium levels at each event (e.g. due to multiple spikes or to adaptation [98]), we sample the overall concentration as coming from a unit log-normal distribution (i.e. an exponentiated Normal distribution $\mathcal{N}(0,1))$.

Once the spike times are obtained, an auto-regressive model with $p$ degrees of freedom (AR- $p$ ) is used to simulate the calcium and fluorescence impulse response. As a difference equation, AR- $p$ models can be written as

$$
y[n]=\sum_{i=1}^{p} a_{i} y[n-i]+b x[n],
$$

where the $a_{i}$ 's are the AR coefficients and $b$ is a scalar multiple of the input. The impulse response can be obtained by solving the inverse Laplace transform

$$
y[n]=h[n] * x[n], \quad h[n]=\mathcal{L}^{-1}\left\{\frac{b z^{p}}{z^{p}-\sum_{i=1}^{p} a_{i} z^{p-i}}\right\} .
$$

Standard linear systems theory shows that $h[n]$ will be composed of the exponentiated roots of the characteristic polynomial $z^{p}-\sum_{i=1}^{p} a_{i} z^{p-i}$, and therefore will be an exponentially decaying function. Higher order polynomials can result in a rise time as well. For this work we find that an AR-2 model $(p=2)$ sufficiently models the rise and fall of observed GCAMP responses. The filter $\boldsymbol{h}$ is convolved with the spike-time vector to create the temporal activity per neuron.

\subsection{3 $\left[\mathrm{Ca}^{2+}\right]$ dynamics}

The fluorescence of a cell is dependent on the number of calcium ions bound to the indicator. if we denote $\left[\mathrm{Ca}^{2+}\right]$ as the amount of free calcium in the cell and [B] as the number of proteins in the cell, we can use the binding/unbinding dynamics, coupled with the entry/exit dynamics of $\left[\mathrm{Ca}^{2+}\right]$ in the cell to determine the fluorescence level at any given time. Specifically, we use the nonlinear diffusion of $\left[\mathrm{Ca}^{2+}\right]$

$$
\frac{\mathrm{d}\left[\mathrm{Ca}^{2+}\right]}{\mathrm{d} t}=-\gamma\left(1+\kappa_{s}+\frac{[\mathrm{B}] K_{d}}{\left(\left[\mathrm{Ca}^{2+}\right]+K_{d}\right)^{2}}\right)^{-1}\left(\left[\mathrm{Ca}^{2+}\right]-\left[\mathrm{Ca}^{2+}\right]_{r e s t}\right),
$$


where $\left[\mathrm{Ca}^{2+}\right]_{\text {rest }}$ represents the baseline free $\left[\mathrm{Ca}^{2+}\right], \gamma$ is the $\left[\mathrm{Ca}^{2+}\right]$ diffusion constant, $\kappa_{s}$ is the endogenous $\left[\mathrm{Ca}^{2+}\right]$ binding ratio, and $K_{d}$ is the protein binding affinity constant [67,68]. As $\gamma$ is a function of the volume-to-surface area, we use a different $\gamma$ value for dendrite dynamics as for dynamics in the soma [99]. While this model permits simulation of the $\left[\mathrm{Ca}^{2+}\right]$ concentration over time, the model does not include the on/off time constants $\tau_{\text {on }}$ and $\tau_{\text {off }}$ that describe how long it takes for the bound proteins to become active. We can model this effect, as in [68], by convolving with a double-exponential function

$$
h(t)=A\left(1-e^{-t / \tau_{o n}}\right) e^{-t / \tau_{o f f}}
$$

where the amplitude $A$ and the time constants $\tau_{\text {on }}$ and $\tau_{\text {off }}$ can be fit to the particular protein kinetics. The final step in simulating the fluorescence time-traces is to convert the calcium concentrations to fluorescence levels. For this task, we use the Hill equation

$$
\Delta F / F=\frac{1}{1+\left(K_{D} /\left[\mathrm{Ca}^{2+}\right]\right)^{n_{H}}}
$$

where the parameters $K_{D}$ and $n_{H}$ have been measured in the literature (specifically [70], Table 1), and the absolute florescence is

$$
F=F_{0}(\Delta F / F)+F_{0}
$$

where the baseline fluorescence $F_{0}$ can be tuned to the protein statistics.

\subsection{Optics simulation}

The optics module consists of modeling the shape and intensity of the point-spread function (PSF) within the scanned tissue. For computational purposes, we assume the shape of the PSF is constant across the scanned volume and only the amplitude is modulated. We estimate the PSF within the scanned tissue by propagating a specified field through the simulated tissue across the field of view.

We describe the scalar field at the front aperture of the objective lens as a Gaussian with a circular aperture and spherical phase:

$$
U_{0}(\rho)= \begin{cases}\exp \left(-\frac{\rho^{2}}{\rho_{e}^{2}}-\frac{i k \rho^{2}}{2 f}\right) & \rho \leqslant \rho_{0} \\ 0 & \rho>\rho_{0}\end{cases}
$$

where $U_{0}$ is the scalar field, $\rho=\sqrt{x^{2}+y^{2}}$ is the polar position, $k$ is the wavenumber, $\rho_{0}$ is the radius of the objective lens, $\rho_{e}$ is the radius of the excitation beam, and $f$ is the focal length of the objective lens. The wavefront is multiplied by any additional specified aberrations due to the microscope or the sample:

$$
U(\rho, \theta)=U_{0}(\rho) \exp \left(i k \sum_{i=1}^{\infty} a_{i} Z_{i}(\rho, \theta)\right)
$$

where $\theta$ is the polar angle, $a_{i}$ are the Zernike coefficients, and $Z_{i}$ are the Zernike polynomials. By default, only spherical aberration approximating the contribution of the refractive index mismatch of the sample and astigmatism approximating the contribution of offset scanning galvanometers are included. 
The field $U(\rho, \theta)$ is propagated through the sample to the focal plane along a $2 \mathrm{D}$ grid of positions within a simulated refractive index volume $\delta n$. The volume $\delta n$ is generated from the simulated vasculature and a 3D Gaussian process with a weight distribution approximating the refractive index distribution of mouse cortical tissue (see Supp Fig. 10) [100]:

$$
\delta n(x, y, z)=n_{\text {diff }}(V(x, y, z)+G P(x, y, z))
$$

where $V$ is the vasculature and $G P$ is the smooth Gaussian Process representing the optical properties in the non-vasculature areas. The vasculature provides the bulk of the long range refractive index shifts in the simulation, while the Gaussian process approximates the local shifts.

The Fresnel diffraction integral is used to estimate the field throughout the volume, and the split-step beam propagation method [101] is used to apply the effects of inhomogeneity within the volume. The simulated phase-difference volume is summed into optical phase masks corresponding to each propagation step:

$$
\phi\left(x_{i}, y_{i}, z_{i}\right)=k \int_{z_{i}}^{z_{i+1}} \delta n(x, y, z) d z .
$$

This quantity is multiplied after each optical propgation step as

$$
U_{i+1}=\frac{e^{i k z}}{i \lambda\left(z_{i+1}-z_{i}\right)} \int_{\infty}^{\infty} \int_{-\infty}^{\infty} e^{-i \phi_{i}} U_{i} e^{\frac{i k}{2\left(z_{i+1}-z_{i}\right)}\left[\left(x_{i+1}-x_{i}\right)^{2}+\left(y_{i+1}-y_{i}\right)^{2}\right]} d x_{i} d y_{i},
$$

where $\phi_{i}=\phi\left(x_{i}, y_{i}, z_{i}\right)$ is the optical phase mask and $U_{i}=U\left(x_{i}, y_{i}, z_{i}\right)$ is the scalar field at each position. The resultant 3D field generated by the propagation is then used to calculate the twophoton PSF:

$$
P S F(x, y, z)=U(x, y, z)^{4} .
$$

The aberrations caused by the phase differences approximate the effects of wavefront distortions caused by refractive index inhomogeneity within the imaged sample [102, 103]. The two-photon PSFs at each location across the field of view are averaged to obtain the PSF to be scanned through the simulation, and the summed intensity of the PSFs across the field are used to generate an intensity scaling mask for scanning. For runtime considerations, the PSF near the focal plane is sampled at the resolution of the volume while the out of focus PSF and scaling mask is sampled at a reduced resolution.

For alternative optical setups, we adjust the input field $U_{0}$ accordingly. For a low numerical aperture excitation beam, $\rho_{e}$ is reduced, and for a Bessel beam excitation $U_{0}$ is replaced with an excitation ring. See supplementary information for more details.

An additional optical mask is also calculated by estimating the reduction in signal from absorption of the collected light by the vasculature. The collected light at each scanned position is reduced by a collection cone corresponding to the simulated collection objective numerical aperture:

$$
r(z)=\tan \left(\sin ^{-1}(N A / n)\right) z
$$

where $r(z)$ is the collected cone radius as a function of depth, and:

$$
C(x, y)=10^{A \int_{0}^{d}(r(z) * V(x, y, z)) d z}
$$

where $C$ is fraction of light collected, $d$ is the tissue depth, and $A$ is the adjusted light absorbance of light emitted from GFP normalized by the arterial blood absorbance factor. This absorbance mask is multiplied to the optical excitation mask to give the combined spatial signal scaling mask. 


\subsection{Scanning in silico}

The final module takes the generated volume, the generated PSF and time-traces, and generates the TPM output frames. The first step here is to use the time-traces and fluorescence distribution for each neuron to "color in" the corresponding volume with the current fluorescence level for that neuron. Similarly, the background level is set by repeating this process with the neuropil. The PSF is then convolved with the current volume, and the result is masked with the optical path mask to create an initial image.

To simulate motion in the movie, we select a portion of this initial frame to treat as the entire image. The starting position (upper left corner) for the with-motion frame is moved according to a small $+/-0.5 \mu-\mathrm{m}$ jitter with occasional larger jumps (up to $2-3 \mu \mathrm{m}$ ). Options to include per-line motion and shearing are also implemented by choosing different sub-sections of each row as the withmotion frame is extracted from the larger motionless frame. This frame represents the fluorescence level at each point in the sampled image. To obtain the actual electrical signals sampled by the TPM device, we apply a noise model that simulates the number of photons incident on the array (modeled as Poisson) followed by an electrical noise model that is Gaussian, with increasing mean and variance with larger numbers of incident photons. If $\lambda$ is the true florescence for a pixel, $x$ is the number of incident photons, and $y$ is the measured electrical signal, the noise model can be expressed as

$$
\begin{aligned}
& x \sim \operatorname{Poisson}(\lambda) \\
& y \sim \log \mathcal{N}\left(\mu_{0}+\mu x, \sigma_{0}^{2}+\sigma^{2} x\right)
\end{aligned}
$$

where $\mu_{0}$ and $\sigma_{0}^{3}$ are the baseline noise mean and variance (with no photons), and $\mu$ and $\sigma^{2}$ are the parameters controlling how the measurement mean and variance grow with increased incident photons.

As a final step, we simulate the analog-to-digital accumulators' property where photons arriving in one pixel's accumulation time can cause an analog shape that bleeds through to the accumulation for the next pixel (Fig. 12). We simulate this effect by noting that if a photon arrives early in the sample period, then the analog PMT response $g(t)$ is completely inside of the sample period and no bleed-through occurs. On the other hand, if the photon arrives within $\Delta$ of the end of the sampling period, where $\Delta$ is the temporal extent of $g(t)$ (Fig. 12), then the tail end of $g(t)$ that continues beyond the end of the period is integrated into the next sample. The probability of a given bleed-through level for one photon can thus be quantified as

$$
p(b)=\left\{\begin{array}{ccc}
1-\frac{\Delta}{T} & \text { for } & b=0 \\
\frac{\Delta}{T} \frac{1}{g(T-\tau(b))} & \text { for } & 0<b \leqslant \int_{0}^{\Delta} g(t) \mathrm{d} t
\end{array}\right.
$$

where $\tau(b)$ represents the delay $\tau$ that is needed to result in a given bleed-through $b$. Since the relationship between $b$ and $\tau$,

$$
b=\int_{T-\tau}^{\Delta} g(t) \mathrm{d} t
$$

is monotonically increasing when $g(t) \geqslant 0$ for all $t, \tau(b)$ is a well defined function. Since photon arrivals are approximately independent, the bleed-through probability distribution for multiple photons is the convolution of the distribution for a single photon. The resulting statistical model then 
takes a random fraction (uniformly chosen between zero and 50\%) of each pixel with probability 0.2 , and adds that amount to the next pixel,

$$
p(b)=\left\{\begin{array}{ccc}
1-p_{\text {bleed }} & \text { for } & b=0 \\
\frac{p_{\text {bleed }}}{b_{\text {max }}} & \text { for } & 0<b \leqslant b_{\text {max }}
\end{array}\right.
$$

\section{Hemoglobin absorption}

To calculate the absorption due to hemoglobin, we assume default concentrations pf $150 \mathrm{mg} / \mathrm{ml} \mathrm{Hb}$, $64500 \mathrm{~g} / \mathrm{mol} \mathrm{Hb}, 2.9(\mathrm{abs} / \mu \mathrm{m}) /(\mathrm{mol} / \mathrm{L})$ in units of abs $/ \mu \mathrm{m}$. The absorbance is then calculated using Scott Prahl's Hb curve [104] and eGFP emission spectrum [105].

\section{Estimation of per-trace noise variance}

To estimate the noise variance for each time-trace, we begin with the basic per-pixel noise model

$$
\boldsymbol{y}=\boldsymbol{\Phi} \boldsymbol{f}+\boldsymbol{d}+\boldsymbol{\epsilon}
$$

where the noise is heteroskedastic in that the variance is proportional to the mean

$$
\boldsymbol{\epsilon} \sim \mathcal{N}(\mathbf{0}, \operatorname{diag}(\mathbf{\Phi} \boldsymbol{f}+\boldsymbol{d}))
$$

The least-squares estimate of the activations under the imperfect spatial profiles $\widehat{\boldsymbol{\Phi}}$ is

$$
\begin{aligned}
& \hat{\boldsymbol{f}}=\arg \min _{\boldsymbol{f}}\|\boldsymbol{y}-\hat{\boldsymbol{\Phi}} \boldsymbol{f}\|_{2}^{2} \\
& =\left(\widehat{\boldsymbol{\Phi}}^{T} \widehat{\boldsymbol{\Phi}}\right)^{-1} \hat{\boldsymbol{\Phi}}^{T} \boldsymbol{y} \\
& =\left(\hat{\boldsymbol{\Phi}}^{T} \hat{\boldsymbol{\Phi}}\right)^{-1} \hat{\boldsymbol{\Phi}}^{T}(\boldsymbol{\Phi} \boldsymbol{f}+\boldsymbol{d}+\boldsymbol{\epsilon})
\end{aligned}
$$

The covariance of this estimate is then

$$
\begin{aligned}
\operatorname{Cov}(\widehat{\boldsymbol{f}}) & =\operatorname{Cov}\left[\left(\widehat{\boldsymbol{\Phi}}^{T} \widehat{\boldsymbol{\Phi}}\right)^{-1} \widehat{\boldsymbol{\Phi}}^{T}(\boldsymbol{\Phi} \boldsymbol{f}+\boldsymbol{d}+\boldsymbol{\epsilon})\right] \\
& =\operatorname{Cov}\left[\left(\widehat{\boldsymbol{\Phi}}^{T} \widehat{\boldsymbol{\Phi}}\right)^{-1} \widehat{\boldsymbol{\Phi}}^{T} \boldsymbol{\epsilon}\right] \\
& =E\left[\left(\widehat{\boldsymbol{\Phi}}^{T} \widehat{\boldsymbol{\Phi}}\right)^{-1} \widehat{\boldsymbol{\Phi}}^{T} \boldsymbol{\epsilon} \boldsymbol{\epsilon}^{T} \widehat{\boldsymbol{\Phi}}\left(\widehat{\boldsymbol{\Phi}}^{T} \widehat{\boldsymbol{\Phi}}\right)^{-1}\right] \\
& =\left(\widehat{\boldsymbol{\Phi}}^{T} \widehat{\boldsymbol{\Phi}}\right)^{-1} \widehat{\boldsymbol{\Phi}}^{T} E\left[\boldsymbol{\epsilon} \boldsymbol{\epsilon}^{T}\right] \widehat{\boldsymbol{\Phi}}\left(\widehat{\boldsymbol{\Phi}}^{T} \widehat{\boldsymbol{\Phi}}\right)^{-1} \\
& =\left(\widehat{\boldsymbol{\Phi}}^{T} \widehat{\boldsymbol{\Phi}}\right)^{-1} \widehat{\boldsymbol{\Phi}}^{T} \operatorname{diag}(\boldsymbol{\Phi} \boldsymbol{f}+\boldsymbol{d}) \widehat{\boldsymbol{\Phi}}\left(\widehat{\boldsymbol{\Phi}}^{T} \widehat{\boldsymbol{\Phi}}\right)^{-1}
\end{aligned}
$$

\section{Local correlation calculation}

To calculate the local correlations, V1 two-photon recordings and simulations were motion corrected using correlation-based rigid motion correction [106]. A 1500-frame subsection of each dataset over a $250 \times 250$ pixel area was extracted. For each pixel, the Pearson correlation between it's fluorescence activity and that of each of the neighboring pixels in a $51 \times 51$ pixel square neighborhood (up to 25 pixels away in each direction) were calculated. The results were averaged over all pixels (to create the mean images) and histograms were created to depict the spread for correlations along the fast-scan direction. 


\section{Calculation of auxiliary time-traces}

To calculate the auxiliary, noisy "ground truth" time traces, we consider the movie frames $\boldsymbol{y}_{t}$ for $t=1 \ldots T$ and the calculated ground-truth spatial profiles $\boldsymbol{X}=\left[\boldsymbol{x}_{1}, \ldots, \boldsymbol{x}_{N}\right]$. The noisy time trace estimates are then calculated via the least-squares estimation procedure at each time-step $t$

$$
\widehat{s}_{t}=\arg \min _{S}\left\|\boldsymbol{y}_{t}-\boldsymbol{X} \boldsymbol{s}\right\|_{2}^{2} .
$$

\section{Acknowledgements}

The authors would like to thank Thomas Macrina and Sebastian Seung for helpful discussions of anatomy and electron microscopy data, and Chris Schaffer and Mohammad Haft-Javaherian for the use of and helpful discussions pertaining to their vasculature data. Moreover, the authors would like to thank Ben Scott, Sam Wang, Sue Ann Koay, Brian DePasquale and Dylan Rich For their insightful thoughts and comments throughout this project. A.S.C. and J.W.P. were supported by Simons Collaboration on the Global Brain (SCGB AWD543027), and a U19 NIH-NINDS BRAIN Initiative Award (5U19NS104648). A.S. was supported by NIH grant 1U19NS104648. D.W.T. was supported by NIH grants R01MH083868 and 1U19NS104648, and the Simons Collaboration on the Global Brain (SCGB 543051).

\section{Author Contributions}

A.S.C. and A.S. designed and coded the bulk of the simulation software and performed the ensuing analysis. J.L.G. helped design the scanning noise model. A.S.C., A.S., J.W.P. and D.W.T. wrote the manuscript. J.W.P. and D.W.T. supervised the project.

\section{Competing Financial Interests}

The authors declare no competing financial interests. 


\section{References}

1. Ogawa, S., Lee, T.-M., Kay, A. R. \& Tank, D. W. Brain magnetic resonance imaging with contrast dependent on blood oxygenation. Proceedings of the National Academy of Sciences 87, 9868-9872 (1990).

2. Jun, J. J. et al. Fully integrated silicon probes for high-density recording of neural activity. Nature 551, 232 (2017).

3. Macé, E. et al. Functional ultrasound imaging of the brain. Nature Methods 8, 662 (2011).

4. Kodandaramaiah, S. B., Franzesi, G. T., Chow, B. Y., Boyden, E. S. \& Forest, C. R. Automated whole-cell patch-clamp electrophysiology of neurons in vivo. Nature methods $\mathbf{9}, 585$ (2012).

5. Briggman, K. L., Helmstaedter, M. \& Denk, W. Wiring specificity in the direction-selectivity circuit of the retina. Nature 471, 183 (2011).

6. Bock, D. D. et al. Network anatomy and in vivo physiology of visual cortical neurons. Nature 471, 177 (2011).

7. Yuste, R. \& Denk, W. Dendritic spines as basic functional units of neuronal integration. Nature 375, 682 (1995).

8. Lu, R. et al. Video-rate volumetric functional imaging of the brain at synaptic resolution. Nature neuroscience 20, 620 (2017).

9. Grinvald, A., Lieke, E., Frostig, R. D., Gilbert, C. D. \& Wiesel, T. N. Functional architecture of cortex revealed by optical imaging of intrinsic signals. Nature 324, 361 (1986).

10. Scott, B. B. et al. Imaging cortical dynamics in GCaMP transgenic rats with a head-mounted widefield macroscope. Neuron 100, 1045-1058 (2018).

11. Grienberger, C. \& Konnerth, A. Imaging Calcium in Neurons. Neuron 73, 862-885 (2015).

12. Denk, W., Strickler, J. H. \& Webb, W. W. Two-photon laser scanning fluorescence microscopy. Science 248, 73-6 (1990).

13. Botcherby, E. J., Juskaitis, R. \& Wilson, T. Scanning two photon fluorescence microscopy with extended depth of field. Optics Communications 268, 253-260 (2006).

14. Tian, L., Akerboom, J., Schreiter, E. R. \& Looger, L. L. Neural activity imaging with genetically encoded calcium indicators. Progress in Brain Research 196, 79-94 (2012).

15. Helmchen, F. \& Denk, W. Deep tissue two-photon microscopy. Nature Methods 2, 932-940 (2005).

16. Stosiek, C., Garaschuk, O., Holthoff, K. \& Konnerth, A. In vivo two-photon calcium imaging of neuronal networks. Proceedings of the National Academy of Sciences 100, 7319-7324 (2003).

17. Weisenburger, S. et al. Volumetric Ca2+ Imaging in the Mouse Brain Using Hybrid Multiplexed Sculpted Light Microscopy. Cell 177, 1050-1066 (2019).

18. Pachitariu, M. et al. Suite2p: beyond 10,000 neurons with standard two-photon microscopy. bioRxiv. doi:10.1101/061507. eprint: https://www.biorxiv.org/content/early/2017/07/ 20/061507.full.pdf. <https://www. biorxiv.org/content/early/2017/07/20/061507> (2017). 
19. Harvey, C. D., Collman, F., Dombeck, D. A. \& Tank, D. W. Intracellular dynamics of hippocampal place cells during virtual navigation. Nature 461, 941-6 (2009).

20. Rickgauer, J. P., Deisseroth, K. \& Tank, D. W. Simultaneous cellular-resolution optical perturbation and imaging of place cell firing fields. Nat Neurosci 17, 1816-24 (2014).

21. Dombeck, D. A., Khabbaz, A. N., Collman, F., Adelman, T. L. \& Tank, D. W. Imaging large-scale neural activity with cellular resolution in awake, mobile mice. Neuron 56, 43-57 (2007).

22. Pnevmatikakis, E. A. Analysis pipelines for calcium imaging data. Current opinion in neurobiology 55, 15-21 (2019).

23. Stringer, C. \& Pachitariu, M. Computational processing of neural recordings from calcium imaging data. Current opinion in neurobiology 55, 22-31 (2019).

24. Gauthier, J. L., Tank, D. W., Pillow, J. W. \& Charles, A. S. Detecting and Correcting False Transients in Calcium Time-trace Inference. Submitted. <https : / /ww . biorxiv . org / content/early/2018/11/19/473470> (2018).

25. Rueckel, M., Mack-Bucher, J. A. \& Denk, W. Adaptive wavefront correction in two-photon microscopy using coherence-gated wavefront sensing. Proceedings of the National Academy of Sciences 103, 17137-17142 (2006).

26. Ji, N., Sato, T. R. \& Betzig, E. Characterization and adaptive optical correction of aberrations during in vivo imaging in the mouse cortex. Proceedings of the National Academy of Sciences 109, 22-27 (2012).

27. Svoboda, K., Denk, W., Kleinfeld, D. \& Tank, D. W. In vivo dendritic calcium dynamics in neocortical pyramidal neurons. Nature 385, 161 (1997).

28. Murayama, M. et al. Dendritic encoding of sensory stimuli controlled by deep cortical interneurons. Nature 457, 1137 (2009).

29. Mukamel, E. A., Nimmerjahn, A. \& Schnitzer, M. J. Automated analysis of cellular signals from large-scale calcium imaging data. Neuron 63, 747-760 (2009).

30. Maruyama, R. et al. Detecting cells using non-negative matrix factorization on calcium imaging data. Neural Networks 55, 11-19 (2014).

31. Pnevmatikakis, E., Merel, J., Pakman, A. \& Paninski, L. Bayesian spike inference from calcium imaging data. Asilomar Conference on Signals, Systems and Computers, 349-353 (2013).

32. Vogelstein, J. T. et al. Spike inference from calcium imaging using sequential Monte Carlo methods. Biophysical journal 97, 636-655 (2009).

33. Yaksi, E. \& Friedrich, R. W. Reconstruction of firing rate changes across neuronal populations by temporally deconvolved Ca2+ imaging. Nature Methods 3, 377-383 (2006).

34. Pachitariu, M. et al. Extracting regions of interest from biological images with convolutional sparse block coding. Advances in Neural Information Processing Systems, 1745-1753 (2013).

35. Apthorpe, N. et al. Automatic neuron detection in calcium imaging data using convolutional networks in Advances in Neural Information Processing Systems (2016), 3270-3278.

36. Jon, O., Schultz, S. R., Dragotti, P. L., et al. A finite rate of innovation algorithm for fast and accurate spike detection from two-photon calcium imaging. Journal of neural engineering 10, 046017 (2013). 
37. Pnevmatikakis, E. \& Paninski, L. Sparse nonnegative deconvolution for compressive calcium imaging: algorithms and phase transitions. Advances in Neural Information Processing Systems, 1250-1258 (2013).

38. Pnevmatikakis, E. et al. A structured matrix factorization framework for large scale calcium imaging data analysis. arXiv preprint arXiv:1409.2903 (2014).

39. Petersen, A., Simon, N. \& Witten, D. SCALPEL: Extracting Neurons from Calcium Imaging Data. arXiv preprint arXiv:1703.06946 (2017).

40. Reynolds, S. et al. ABLE: an activity-based level set segmentation algorithm for two-photon calcium imaging data. ENeuro 4 (2017).

41. Inan, H., Erdogdu, M. A. \& Schnitzer, M. Robust estimation of neural signals in calcium imaging in Advances in Neural Information Processing Systems (2017), 2901-2910.

42. Mishne, G., Coifman, R. R., Lavzin, M. \& Schiller, J. Automated cellular structure extraction in biological images with applications to calcium imaging data. bioRxiv, 313981 (2018).

43. Mishne, G. \& Charles, A. S. Learning Spatially-corellated Temporal Dictionaries for Calcium Imaging. Proceedings of the IEEE International Conference on Acoustics, Speech, and Signal Processing (ICASSP), Brighton, UK (May 2019).

44. Theis, L. et al. Supervised learning sets benchmark for robust spike detection from calcium imaging signals. arXiv preprint arXiv:1503.00135 (2015).

45. Prada, J. et al. An open source tool for automatic spatiotemporal assessment of calcium transients and local 'signal-close-to-noise'activity in calcium imaging data. PLoS computational biology 14, e1006054 (2018).

46. Chen, T.-W. et al. Ultrasensitive fluorescent proteins for imaging neuronal activity. Nature 499, 295-300 (2013).

47. Theis, L. et al. Benchmarking spike rate inference in population calcium imaging. Neuron 90, 471-482 (2016).

48. Berens, P. et al. Standardizing and benchmarking data analysis for calcium imaging. Computational and Systems Neuroscience (2017).

49. Song, A. et al. Volumetric two-photon imaging of neurons using stereoscopy (vTwINS). Nature methods 14, 420 (2017).

50. Nöbauer, T. et al. Video rate volumetric Ca $2+$ imaging across cortex using seeded iterative demixing (SID) microscopy. Nature Methods 14, 811 (2017).

51. Welvaert, M., Durnez, J., Moerkerke, B., Verdoolaege, G. \& Rosseel, Y. neuRosim: An R package for generating fMRI data. Journal of Statistical Software 44, 1-18 (2011).

52. Pnevmatikakis, E. A. et al. Simultaneous denoising, deconvolution, and demixing of calcium imaging data. Neuron 89, 285-299 (2016).

53. Weisenburger, S., Prevedel, R. \& Vaziri, A. Quantitative evaluation of two-photon calcium imaging modalities for high-speed volumetric calcium imaging in scattering brain tissue. bioRxiv, 115659 (2017).

54. Abdellah, M. et al. Bio-physically plausible visualization of highly scattering fluorescent neocortical models for in silico experimentation. BMC bioinformatics 18, 62 (2017). 
55. Markram, H. et al. Reconstruction and simulation of neocortical microcircuitry. Cell 163, 456-492 (2015).

56. Blinder, P. et al. The cortical angiome: an interconnected vascular network with noncolumnar patterns of blood flow. Nature neuroscience 16, 889-897 (2013).

57. Xu, L. \& Mould, D. in Artificial Intelligence Techniques for Computer Graphics 83-102 (2009).

58. Braitenberg, V. \& Schüz, A. Cortex: Statistics and Geometry of Neuronal Connectivity ISBN: 9783540638162 (Springer, 1998).

59. Jiang, X. et al. Principles of connectivity among morphologically defined cell types in adult neocortex. Science 350, aac9462 (2015).

60. Allen cell types database - overview. technical report. Allen Brain Institute. $<$ http://help. brain-map . org/download/attachments /8323525/CellTypesOverview . pdf ? version=1\& modificationDate $=1456188760121>(2015)$.

61. Benavides-Piccione, R. et al. On dendrites in Down syndrome and DS murine models: a spiny way to learn. Progress in neurobiology 74, 111-126 (2004).

62. Watts, D. J. \& Strogatz, S. H. Collective dynamics of 'small-world'networks. nature 393, 440 (1998).

63. Downes, J. H. et al. Emergence of a small-world functional network in cultured neurons. PLoS computational biology 8, e1002522 (2012).

64. Guzman, S. J., Schlögl, A., Frotscher, M. \& Jonas, P. Synaptic mechanisms of pattern completion in the hippocampal CA3 network. Science 353, 1117-1123 (2016).

65. Schroeter, M. S., Charlesworth, P., Kitzbichler, M. G., Paulsen, O. \& Bullmore, E. T. Emergence of rich-club topology and coordinated dynamics in development of hippocampal functional networks in vitro. Journal of Neuroscience 35, 5459-5470 (2015).

66. Greenberg, D. S. et al. Accurate action potential inference from a calcium sensor protein through biophysical modeling. BioRxiv, 479055 (2018).

67. Helmchen, F. \& Tank, D. W. A Single-Compartment Model of Calcium Dynamics in Nerve Terminals and Dendrites. Cold Spring Harbor Protocols 2015, pdb-top085910 (2015).

68. Lutcke, H., Gerhard, F., Zenke, F., Gerstner, W. \& Helmchen, F. Inference of neuronal network spike dynamics and topology from calcium imaging data. Frontiers in Neural Circuits 7, 201. ISSN: 1662-5110 (2013).

69. Deneux, T. et al. Accurate spike estimation from noisy calcium signals for ultrafast threedimensional imaging of large neuronal populations in vivo. Nature communications 7, 12190 (2016).

70. Badura, A., Sun, X. R., Giovannucci, A., Lynch, L. A. \& Wang, S. S.-H. Fast calcium sensor proteins for monitoring neural activity. Neurophotonics 1, 025008-025008 (2014).

71. Sun, X. R. et al. Fast GCaMPs for improved tracking of neuronal activity. Nature communications 4 (2013).

72. Zipfel, W. R., Williams, R. M. \& Webb, W. W. Nonlinear magic: multiphoton microscopy in the biosciences. Nature biotechnology 21, 1369 (2003). 
73. Thériault, G., Cottet, M., Castonguay, A., McCarthy, N. \& De Koninck, Y. Extended twophoton microscopy in live samples with Bessel beams: steadier focus, faster volume scans, and simpler stereoscopic imaging. Frontiers in cellular neuroscience 8 (2014).

74. Tsai, P. S. et al. Correlations of neuronal and microvascular densities in murine cortex revealed by direct counting and colocalization of nuclei and vessels. Journal of Neuroscience 29, 1455314570 (2009).

75. Pathak, A. P., Kim, E., Zhang, J. \& Jones, M. V. Three-dimensional imaging of the mouse neurovasculature with magnetic resonance microscopy. PloS one 6, e22643 (2011).

76. Hrabětová, S. \& Nicholson, C. Biophysical properties of brain extracellular space explored with ion-selective microelectrodes, integrative optical imaging and related techniques (2007).

77. Lee, W.-C. A. et al. Anatomy and function of an excitatory network in the visual cortex. Nature 532, 370 (2016).

78. Stuart, G., Spruston, N. \& Häusser, M. Dendrites (Oxford University Press, 2016).

79. Louth, E. L., Luctkar, H. D., Heney, K. A. \& Bailey, C. D. Developmental ethanol exposure alters the morphology of mouse prefrontal neurons in a layer-specific manner. Brain research 1678, 94-105 (2018).

80. Koester, H. J. \& Sakmann, B. Calcium dynamics associated with action potentials in single nerve terminals of pyramidal cells in layer $2 / 3$ of the young rat neocortex. The Journal of Physiology 529, 625-646 (2000).

81. Kaiser, K. M., Zilberter, Y. \& Sakmann, B. Back-propagating action potentials mediate calcium signalling in dendrites of bitufted interneurons in layer $2 / 3$ of rat somatosensory cortex. The Journal of Physiology 535, 17-31 (2001).

82. Helmchen, F., Imoto, K. \& Sakmann, B. Ca2+ buffering and action potential-evoked Ca2+ signaling in dendrites of pyramidal neurons. Biophysical Journal 70, 1069-1081 (1996).

83. Cox, C. L., Denk, W., Tank, D. W. \& Svoboda, K. Action potentials reliably invade axonal arbors of rat neocortical neurons. Proceedings of the National Academy of Sciences 97, 97249728 (2000).

84. Zariwala, H. A. et al. A Cre-dependent GCaMP3 reporter mouse for neuronal imaging in vivo. Journal of Neuroscience 32, 3131-3141 (2012).

85. Huber, D. et al. Multiple dynamic representations in the motor cortex during sensorimotor learning. Nature 484, 473 (2012).

86. Korogod, N., Petersen, C. C. \& Knott, G. W. Ultrastructural analysis of adult mouse neocortex comparing aldehyde perfusion with cryo fixation. Elife 4, e05793 (2015).

87. Grewe, B. F., Voigt, F. F., van’t Hoff, M. \& Helmchen, F. Fast two-layer two-photon imaging of neuronal cell populations using an electrically tunable lens. Biomedical Optics Express 2, 2035-2046 (2011).

88. Prevedel, R. et al. Fast volumetric calcium imaging across multiple cortical layers using sculpted light. Nature Methods 13, 1021 (2016).

89. Nguyen, J. P. et al. Whole-brain calcium imaging with cellular resolution in freely behaving Caenorhabditis elegans. Proceedings of the National Academy of Sciences 113, E1074-E1081 (2016). 
90. Akerboom, J. et al. Optimization of a GCaMP calcium indicator for neural activity imaging. Journal of Neuroscience 32, 13819-13840 (2012).

91. Institute, A. The Brain Modeling Toolkit (BMTK) https://github.com/AllenInstitute/ bmtk. 2017.

92. Greenwald, M. Beyond benchmarking-how experiments and simulations can work together in plasma physics. Computer physics communications 164, 1-8 (2004).

93. Kasthuri, N. et al. Saturated reconstruction of a volume of neocortex. Cell 162, 648-661 (2015).

94. Rasmussen, C. E. \& Williams, C. K. I. Gaussian Processes for Machine Learning (Adaptive Computation and Machine Learning) ISBN: 026218253X (The MIT Press, 2005).

95. C. Carlson. How I made wine glasses from sunflowers. <http://blog. wolfram.com/2011/ 07/28/how-i-made-wine-glasses-from-sunflowers/> (2011).

96. Hawkes, A. G. Spectra of some self-exciting and mutually exciting point processes. Biometrika 58, 83-90 (1971).

97. Ogata, Y. On Lewis' simulation method for point processes. IEEE Transactions on Information Theory 27, 23-31 (1981).

98. Roxin, A., Brunel, N., Hansel, D., Mongillo, G. \& van Vreeswijk, C. On the distribution of firing rates in networks of cortical neurons. Journal of Neuroscience 31, 16217-16226 (2011).

99. Holthoff, K., Tsay, D. \& Yuste, R. Calcium dynamics of spines depend on their dendritic location. Neuron 33, 425-437 (2002).

100. Jacques, S. L. Optical properties of biological tissues: a review. Physics in Medicine $\&$ Biology 58, R37 (2013).

101. Schmidt, J. D. Numerical simulation of optical wave propagation with examples in MATLAB (SPIE Bellingham, Washington, USA, 2010).

102. Chaigneau, E., Wright, A. J., Poland, S. P., Girkin, J. M. \& Silver, R. A. Impact of wavefront distortion and scattering on 2-photon microscopy in mammalian brain tissue. Optics express 19, 22755-22774 (2011).

103. Wang, C. \& Ji, N. Pupil-segmentation-based adaptive optical correction of a high-numericalaperture gradient refractive index lens for two-photon fluorescence endoscopy. Optics letters 37, 2001-2003 (2012).

104. Prahl, S. Optical absorption of hemoglobin. http://omlc. ogi. edu/spectra/hemoglobin (1999).

105. Spectrum of eGFP http://www.tsienlab.ucsd.edu/Documents.htm. Accessed: 2019-02-04.

106. Giovannucci, A. et al. CaImAn: An open source tool for scalable Calcium Imaging data Analysis. bioRxiv, 339564 (2018).

107. Rall, W. Branching dendritic trees and motoneuron membrane resistivity. Experimental neurology 1, 491-527 (1959).

108. Sun, J., Lee, S. J., Wu, L., Sarntinoranont, M. \& Xie, H. Refractive index measurement of acute rat brain tissue slices using optical coherence tomography. Optics express 20, 10841095 (2012). 
109. Mütze, J. et al. Excitation spectra and brightness optimization of two-photon excited probes. Biophysical journal 102, 934-944 (2012).

110. Watanabe K., N. C. Microscope Objective Lens. Japanese Patent No. 2005-189732 (2005).

111. Metal Package PMT with Cooler Photosensor Modules H7422 Series. Accessed Feb. 5, 2019. Hamamatsu ().

112. Chameleon Ultra Family Widely Tunable, Hands-Free, Modelocked Ti:Sapphire Lasers Ultra, Ultra I, Ultra II. Coherent (2013).

113. Rademacker, J. An exact formula to describe the amplification process in a photomultiplier tube. Nuclear Instruments and Methods in Physics Research Section A: Accelerators, Spectrometers, Detectors and Associated Equipment 484, 432-443 (2002).

114. Chen, T. W. et al. Ultrasensitive fluorescent proteins for imaging neuronal activity. Nature 499, 295-300 (2013). 


\subsection{Supplementary tables}

\begin{tabular}{|c|c|c|c|c|c|}
\hline \multicolumn{6}{|l|}{ Anatomical: } \\
\hline Parameter & Lit. Val. & Fit type & $\begin{array}{c}\text { NAOMi } \\
\text { Val. }\end{array}$ & Unit & Refs \\
\hline Neural density & $9.20 \mathrm{E}+04$ & Direct & - & $\overline{\mathrm{mm}^{-3}}$ & [58] \\
\hline Axonal diameter & 0.3 & Direct & - & $\mu \mathrm{m}$ & {$[58]$} \\
\hline Fraction vasculature & $0.01-0.04$ & Indirect & 0.032 & - & {$[58,74,75]$} \\
\hline Fraction cell bodies & 0.12 & Indirect & 0.135 & - & {$[58]$} \\
\hline Fraction neuropil & 0.84 & Indirect & 0.833 & - & {$[58]$} \\
\hline Fraction dendrites* & 0.294 & Indirect & 0.223 & - & {$[58]$} \\
\hline Fraction other (fluorescing)* & 0.401 & Indirect & 0.33 & - & [58] \\
\hline Fraction other (not fluorescing)* & 0.293 & Indirect & 0.28 & - & {$[58,76]$} \\
\hline Vessel radius (capillary) & $2.00 \mathrm{E}+00$ & Direct & - & $\mu \mathrm{m}$ & {$[56]$} \\
\hline Vessel radius (penetrating) & $10(9,11)$ & Direct & - & $\mu \mathrm{m}$ & {$[56]$} \\
\hline Vessel radius (surface) & $4-30$ & Direct & - & $\mu \mathrm{m}$ & {$[56]$} \\
\hline Vessel length & 50 & Indirect & 50 & $\mu \mathrm{m}$ & {$[56]$} \\
\hline Vessel orientation & Uniform & Indirect & Uniform & - & {$[56]$} \\
\hline Vascular density & $1-3$ & Indirect & 2 & $\%$ & {$[56,58,74,75]$} \\
\hline Penetrating vessel density & $30^{\dagger}$ & Direct & - & $\mathrm{mm}^{-2}$ & {$[56]$} \\
\hline Somatic volume* & $1.80 \mathrm{E}+03^{\dagger}$ & Indirect & $1.80 \mathrm{E}+03$ & $\mu \mathrm{m}^{3}$ & {$[77]$} \\
\hline Nuclear volume* & $800^{\dagger}$ & Indirect & 800 & $\mu \mathrm{m}^{3}$ & {$[77]$} \\
\hline Cytoplasm volume* & $1000^{\dagger}$ & Indirect & 1000 & $\mu \mathrm{m}^{3}$ & {$[77]$} \\
\hline Somatic radius* & $6.25-7.5^{\dagger}$ & Indirect & 7.5 & $\mu \mathrm{m}$ & {$[60,77]$} \\
\hline Nuclear radius* & $6^{\dagger}$ & Indirect & 6 & $\mu \mathrm{m}$ & {$[77]$} \\
\hline Fraction cell bodies & 0.12 & Indirect & 0.135 & - & [58] \\
\hline Basal dendrite endings & $23-25^{\dagger}$ & Direct & - & - & {$[59-61]$} \\
\hline Basal dendrite diameter & 0.7 & Direct & - & $\mu \mathrm{m}$ & [78] \\
\hline Basal dendrite length & $100-160^{\dagger}$ & Indirect & 105 & $\mu \mathrm{m}$ & [59-61] \\
\hline Apical dendrite endings & $6-20^{\dagger}$ & Direct & - & - & {$[59,60]$} \\
\hline Apical dendrite diameter & $1-2$ & Direct & - & $\mu \mathrm{m}$ & {$[79]$} \\
\hline Rall exponent & 1.5 & Direct & - & - & {$[107]$} \\
\hline
\end{tabular}

Supplementary Table 1: Anatomical parameters used for in-silico simulation of neural activity in layer II/III of mouse primary visual area V1. Values for each parameter were either directly found in the literature or estimated from published data (entries with a $\dagger$ ). The third column indicates whether these parameters were set directly in NAOMi, or were fit indirectly by setting other simulation parameters. In the latter cases, the measured values from a simulated NAOMi volume are shown for comparison, indicating that the simulated anatomy matches measured anatomical statistics. 


\begin{tabular}{|l||c|c|c|c|l|}
\hline \multicolumn{7}{|l|}{ Fluorescence model: } & Anat. Val. & Fit type & $\begin{array}{c}\text { NAOMi } \\
\text { Val. }\end{array}$ & Unit & Refs \\
\hline \hline Parameter & 290 & Direct & - & nMol & {$[70]$} \\
\hline \hline GCaMP6f binding affinity $K_{d}$ & 50.00 & Direct & - & nMol & {$[68]$} \\
\hline Baseline $\mathrm{Ca}^{2+}$ concentration & 2800 & Direct & - & $\mathrm{s}^{-1}$ & {$[67,80,83]$} \\
\hline $\mathrm{Ca}^{2+}$ axon diffusion constant $\gamma$ & 100,110 & Direct & 110 & $\mathrm{AU}$ & {$[67,80-82]$} \\
\hline Endogenouse Ca $^{2+}$ binding ratio $k_{s}$ & 1800 & Data fit & 292.3 & $\mathrm{~s}^{-1}$ & {$[67,80,81]$} \\
\hline $\mathrm{Ca}^{2+}$ diffusion constant $\gamma$ & - & Data fit & 98.62 & $\mathrm{AU}$ & - \\
\hline Onset time constant $\tau_{\text {on }}$ & - & Data fit & 0.85 & $\mathrm{AU}$ & - \\
\hline Decay time constant $\tau_{\text {off }}$ & 2.7 & Direct & - & $\mathrm{AU}$ & {$[70]$} \\
\hline GCaMP6f Hill eqn. exponent $n_{h}$ & 25.2 & Direct & - & $\mathrm{F}$ & {$[70]$} \\
\hline GCaMP6f Hill eqn. amplitude & $10-200$ & Direct & 10 & $\mu \mathrm{M}$ & {$[68,84,85]$} \\
\hline Indicator concentration & & & & & \\
\hline
\end{tabular}

Supplementary Table 2: Fluorescence parameters used for in-silico simulation of neural activity in layer II/III of mouse primary visual area V1. Values for each parameter were either directly found in the literature or estimated from published data (entries with a $\dagger$ ). The third column indicates whether these parameters were set directly, or were fit indirectly by setting other simulation parameters. In the latter cases, the measured values simulated fluorescence traces are shown for comparison, indicating a good match between the NAOMi simulation and known activity statistics.

\subsection{Supplementary figures}
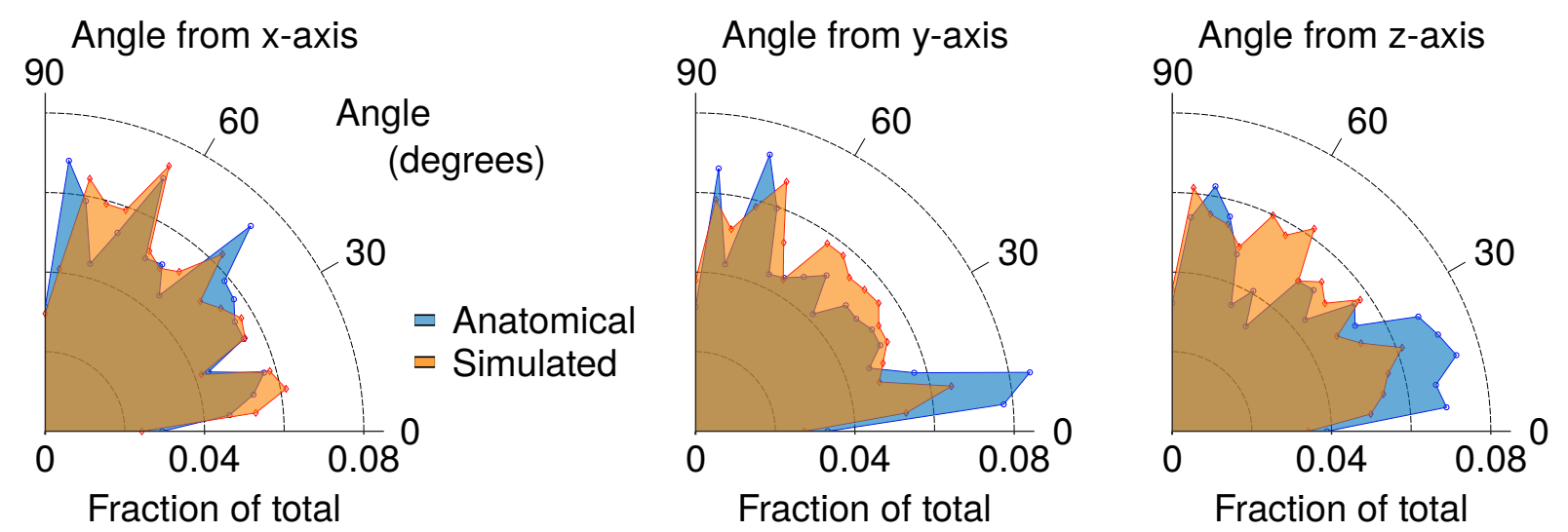

Supplementary Figure 1: Histograms comparing the distribution of vasculature orientation (angles from the $\mathrm{x}-\mathrm{y}$ - and z-axes). 


\begin{tabular}{|c|c|c|c|}
\hline \multicolumn{4}{|l|}{ Optical: } \\
\hline Parameter & Value & Unit & Refs \\
\hline Refractive index (tissue average) & 1.35 & - & {$[108]$} \\
\hline Refractive index (SD) & 0.02 & - & {$[77,108]$} \\
\hline Scatter size & {$\left[\begin{array}{lllll}0.51 & 1.56 & 4.52 & 14.78\end{array}\right]$} & $\mu \mathrm{m}$ & {$[77,100]$, See Methods } \\
\hline Scatter weight & {$\left[\begin{array}{llll}0.57 & 0.29 & 0.19 & 0.15\end{array}\right]$} & - & {$[77,100]$, See Methods } \\
\hline Wavelength $\lambda$ & 920 & $\mathrm{~nm}$ & {$[109]$} \\
\hline System aberrations (astigmatism) & 0.1 & $\lambda$ & See Methods \\
\hline System aberrations (spherical) & 0.12 & $\lambda$ & See Methods \\
\hline Hemoglobin absorption & $0.00674^{*} \log (10)$ & - & [104], See Methods \\
\hline Objective NA & 0.8 & - & [110] \\
\hline PMT QE & 0.4 & - & [111] \\
\hline eGFP Quantum Yield & 0.6 & - & [109] \\
\hline$\Delta$, baseline $F_{0}$ & 2 & GM & {$[109]$} \\
\hline$\Delta$, saturation $F_{\max }$ & 35 & GM & [109] \\
\hline Sech pulse shape & 0.588 & - & {$[112]$} \\
\hline Ti:S rep rate & 80 & $\mathrm{MHz}$ & [112] \\
\hline Ti:S pulse width & 140 & fs & {$[112]$} \\
\hline
\end{tabular}

Supplementary Table 3: Optical parameters used for in-silico simulation of two-photon microscopy scanning.

\begin{tabular}{|l||c|c|l|}
\hline \multicolumn{3}{|l|}{ Scanning: } \\
\hline \hline Parameter & Value & Unit & Refs \\
\hline \hline PMT dynode model & & & {$[113]$, See Methods } \\
\hline PMT Poisson-Gauss & $\begin{array}{c}1,100 \text { (mean photon,electronic) } \\
0.23,2300 \text { (std photon,electronic) }\end{array}$ & counts & See Methods \\
\hline Bleed-through probability & 0.3 & - & See Methods \\
\hline Bleed-through weight & 0.4 & $\mathrm{~V}^{*} \mathrm{~s}$ & See Methods \\
\hline PMT darkcount mean & 0.05 & counts & See Methods \\
\hline
\end{tabular}

Supplementary Table 4: Scanning parameters used for in-silico simulation of two-photon microscopy scanning.
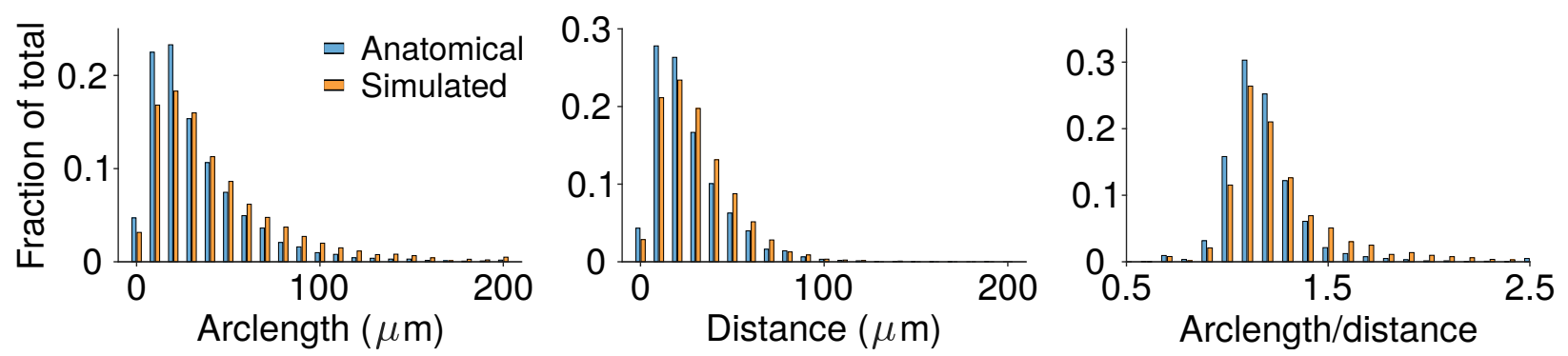

Supplementary Figure 2: Histograms comparing the lengths of vasculature. 


\begin{tabular}{|l|c|c|c|c|l|}
\hline Other parameters: & Anat. Val. & Fit type & $\begin{array}{c}\text { NAOMi } \\
\text { Val. }\end{array}$ & Unit & Refs \\
\hline \hline Parameter & 0.3 & Direct & - & um & {$[58]$} \\
\hline \hline Axonal diameter & $0.01-0.04$ & Indirect & 0.032 & - & {$[58,74,75]$} \\
\hline Fraction vasculature & 0.12 & Indirect & 0.135 & - & {$[58]$} \\
\hline Fraction cell bodies & 0.84 & Indirect & 0.833 & - & {$[58]$} \\
\hline Fraction neuropil & 0.118 & Indirect & 0 & - & {$[58]$} \\
\hline Fraction spines* & 0.294 & Indirect & 0.223 & - & {$[58]$} \\
\hline Fraction dendrites* & 0.283 & Indirect & 0.33 & - & {$[58]$} \\
\hline Fraction Axons* & 0.0924 & Indirect & 0.08 & - & {$[58]$} \\
\hline Fraction Glia* & 0.2 & Direct & 0.2 & - & {$[76]$} \\
\hline Fraction extracellular & \multicolumn{5}{|l|}{ *scaled to full volume [86] } \\
\hline \multicolumn{7}{|l|}{} \\
\hline
\end{tabular}

Supplementary Table 5: Detailed fractional volume of components

\begin{tabular}{|l|c|c|c|c|c|c|c|c|c|}
\hline 40m W power & \multicolumn{3}{|c|}{ CNMF (1091 total) } & \multicolumn{3}{c|}{ Suite2p (661 total) } & \multicolumn{3}{c|}{ PCA/ICA (265 total) } \\
\hline Corr. cutoff & $>0.1$ & $>0.3$ & $>0.5$ & $>0.1$ & $>0.3$ & $>0.5$ & $>0.1$ & $>0.3$ & $>0.5$ \\
\hline Paired & 704 & 522 & 338 & 519 & 479 & 423 & 170 & 152 & 144 \\
\hline Unique & 566 & 438 & 303 & 366 & 335 & 292 & 152 & 139 & 137 \\
\hline Doubled & 95 & 54 & 27 & 68 & 64 & 58 & 11 & 9 & 7 \\
\hline
\end{tabular}

Supplementary Table 6: Results of automated calcium imaging video segmentation as applied to simulated data generated from NAOMi. Of the total number of components isolated, only a fraction $(\approx 30 \%-50 \%)$ unique, true cells in the scanned volume strongly matched the found components.

\begin{tabular}{|l|c|c|c|}
\hline 40m W power & \multicolumn{3}{|c|}{ Unique Cells found } \\
\hline Corr. cutoff & $>0.1$ & $>0.3$ & $>0.5$ \\
\hline CNMF (1091 total) & 566 & 438 & 303 \\
\hline Suite2p (1091 total) & 366 & 335 & 292 \\
\hline PCA/ICA (265 total) & 152 & 139 & 137 \\
\hline CNMF Only & 238 & 69 & 28 \\
\hline Suite2p Only & 43 & 26 & 17 \\
\hline PCA/ICA Only & 13 & 2 & 3 \\
\hline CNMF \& Suite2p & 321 & 307 & 273 \\
\hline CNMF \& PCA/ICA & 144 & 136 & 134 \\
\hline Suite2p \& PCA/ICA & 134 & 137 & 134 \\
\hline All algorithms & 137 & 135 & 132 \\
\hline
\end{tabular}

Supplementary Table 7: Results of automated calcium imaging video segmentation as applied to simulated data generated from NAOMi. While sets of found components largely overlapped between algorithms, each method's design allowed for the extraction of slightly different cell activities. 


\begin{tabular}{|l|c|c|c|}
\hline 40m W power & \multicolumn{3}{|c|}{ Unique Cells found } \\
\hline Corr. cutoff & $>0.1$ & $>0.3$ & $>0.5$ \\
\hline Ideal (8117 total) & 2088 & 817 & 415 \\
\hline Ideal \& not CNMF & 1523 & 383 & 120 \\
\hline Ideal \& not Suite2p & 1722 & 483 & 128 \\
\hline Ideal \& not PCA/ICA & 1936 & 678 & 278 \\
\hline CNMF \& not Ideal & 1 & 4 & 8 \\
\hline Suite2p \& not Ideal & 0 & 1 & 5 \\
\hline PCA/ICA \& not Ideal & 0 & 0 & 0 \\
\hline
\end{tabular}

Supplementary Table 8: Results of automated calcium imaging video segmentation as applied to simulated data generated from NAOMi. De-mixing the data using oracle spatial profile knowledge allowed for isolating many more components, indicating that automated methods have room for improvement. Interestingly some algorithms, due to built in denoising not included in the ideal de-mixing, were able to isolate some cell time-traces more accurately.

\begin{tabular}{|l|c|c|c|c|c|c|c|c|c|}
\hline 80m W power & \multicolumn{3}{|c|}{ CNMF (999 total) } & \multicolumn{3}{c|}{ Suite2p (639 total) } & \multicolumn{3}{c|}{ PCA/ICA (635 total) } \\
\hline Corr. cutoff & $>0.1$ & $>0.3$ & $>0.5$ & $>0.1$ & $>0.3$ & $>0.5$ & $>0.1$ & $>0.3$ & $>0.5$ \\
\hline Paired & 651 & 546 & 460 & 532 & 479 & 462 & 420 & 322 & 292 \\
\hline Unique & 579 & 493 & 424 & 413 & 369 & 358 & 337 & 280 & 264 \\
\hline Doubled & 60 & 41 & 30 & 61 & 54 & 53 & 47 & 28 & 20 \\
\hline
\end{tabular}

Supplementary Table 9: Results of automated calcium imaging video segmentation as applied to simulated data generated from NAOMi with $80 \mathrm{~m}$ W laser power.

\begin{tabular}{|l|c|c|c|}
\hline 80m W power & \multicolumn{3}{|c|}{ Unique Cells found } \\
\hline Corr. cutoff & $>0.1$ & $>0.3$ & $>0.5$ \\
\hline CNMF (999 total) & 579 & 493 & 424 \\
\hline Suite2p (639 total) & 413 & 369 & 358 \\
\hline PCA/ICA (635 total) & 337 & 280 & 264 \\
\hline CNMF Only & 198 & 118 & 85 \\
\hline Suite2p Only & 46 & 24 & 22 \\
\hline PCA/ICA Only & 90 & 38 & 24 \\
\hline CNMF \& Suite2p & 357 & 334 & 325 \\
\hline CNMF \& PCA/ICA & 261 & 247 & 243 \\
\hline Suite2p \& PCA/ICA & 240 & 242 & 240 \\
\hline All algorithms & 237 & 231 & 229 \\
\hline
\end{tabular}

Supplementary Table 10: Results of automated calcium imaging video segmentation as applied to simulated data generated from NAOMi with $80 \mathrm{~m}$ W laser power. 


\begin{tabular}{|l|c|c|c|}
\hline $80 \mathrm{~m}$ W power & \multicolumn{3}{|c|}{ Unique Cells found } \\
\hline Corr. cutoff & $>0.1$ & $>0.3$ & $>0.5$ \\
\hline Ideal (8117 total) & 3206 & 1528 & 904 \\
\hline Ideal \& not CNMF & 2629 & 1036 & 481 \\
\hline Ideal \& not Suite2p & 2793 & 1159 & 546 \\
\hline Ideal \& not PCA/ICA & 2870 & 1248 & 640 \\
\hline CNMF \& not Ideal & 3 & 1 & 1 \\
\hline Suite2p \& not Ideal & 1 & 0 & 0 \\
\hline PCA/ICA \& not Ideal & 2 & 0 & 0 \\
\hline
\end{tabular}

Supplementary Table 11: Results of automated calcium imaging video segmentation as applied to simulated data generated from NAOMi with $80 \mathrm{~m}$ W laser power.

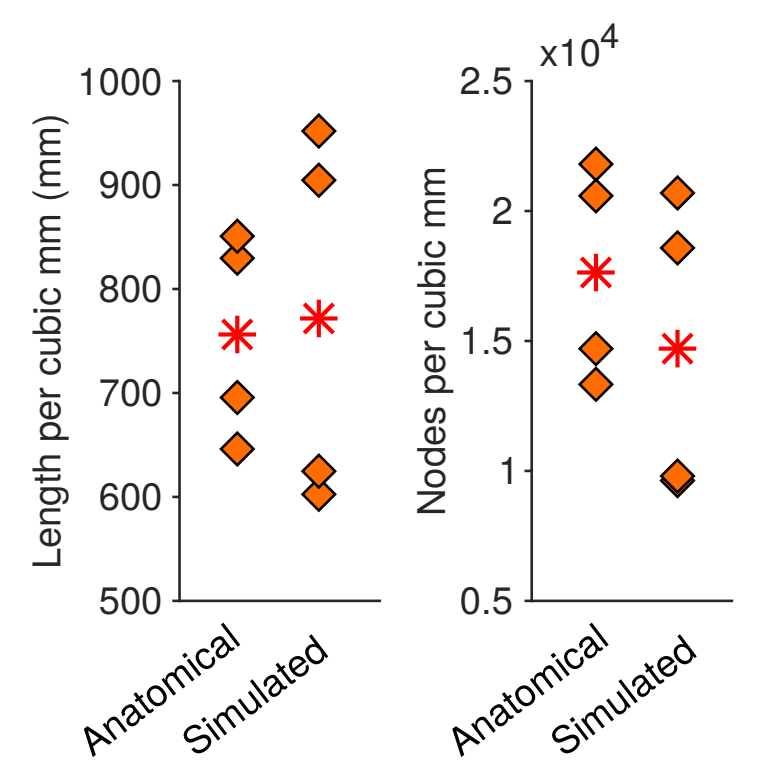

Supplementary Figure 3: Comparison of overall length of the vasculature and the number of vasculature nodes within a cubic mm volume. 
Reconstructed soma and nucleus
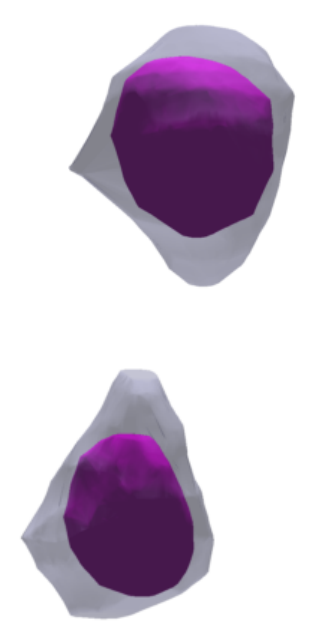
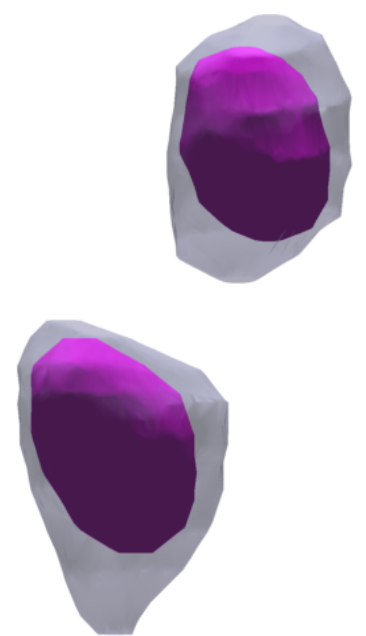

Simulated soma and nucleus
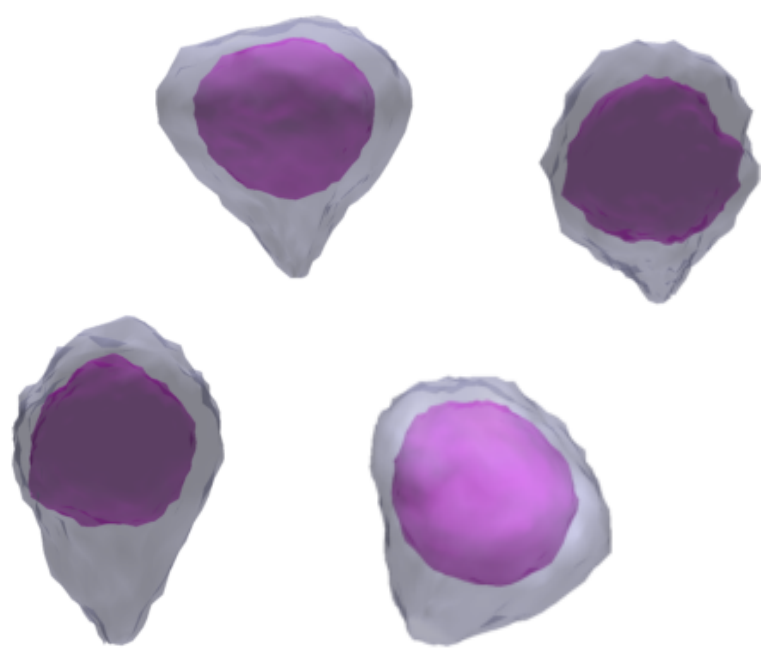

Supplementary Figure 4: Examples of measured and simulated somas. Both measured somas (left) and simulated somas (right) have a bumpy cell wall with one end exhibiting the cone-shaped tightening characteristic of pyramidal cells. The nuclei of both sets of somas (blue shape inside of the red shape) are shrunken and smoothed versions of the exterior cell wall.
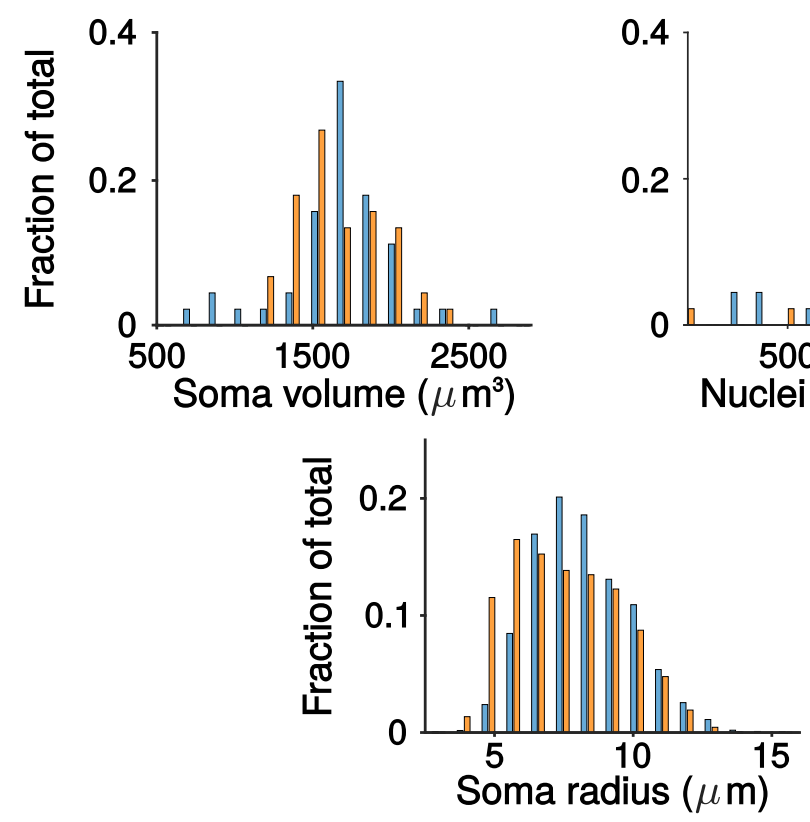
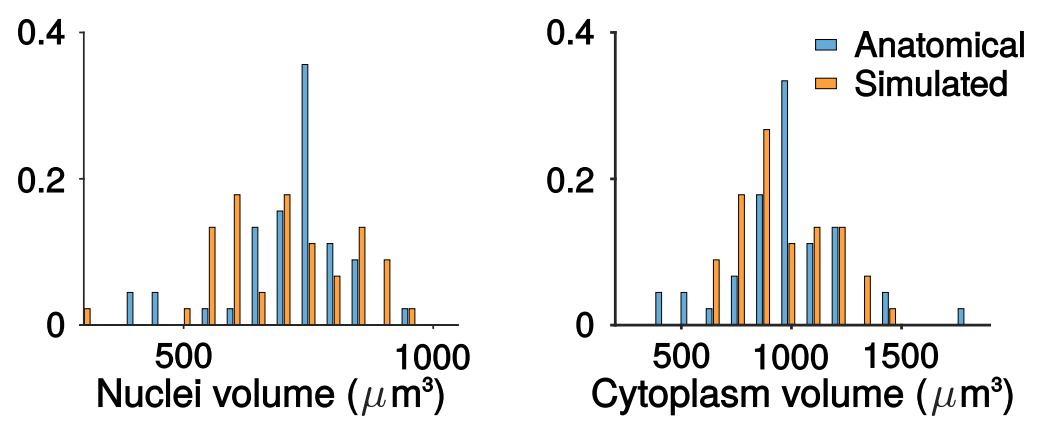

Supplementary Figure 5: Histogram comparing simulated somas with measured anatomy. 

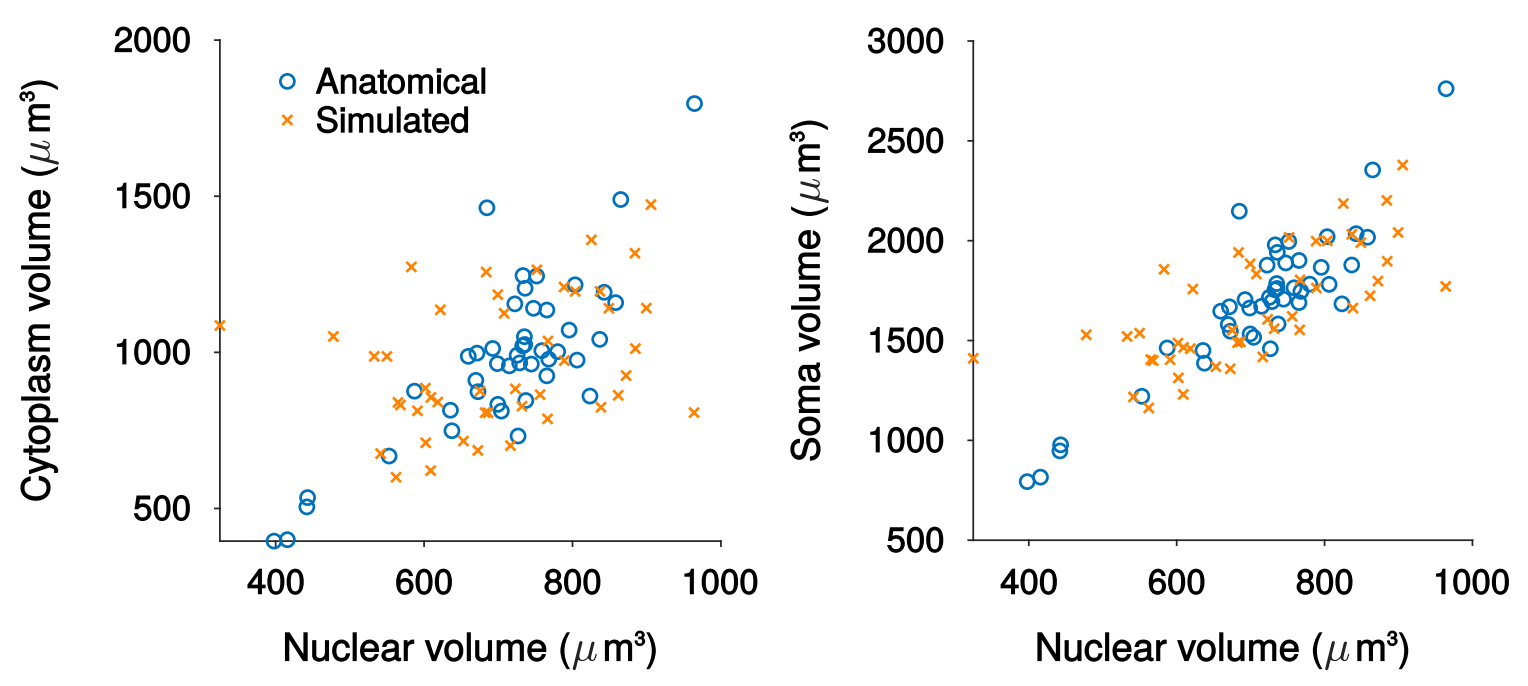

Supplementary Figure 6: Scatter plots comparing the nucleus, soma and cytoplasm volumes for simulated neurons with measured anatomy from EM reconstructions.
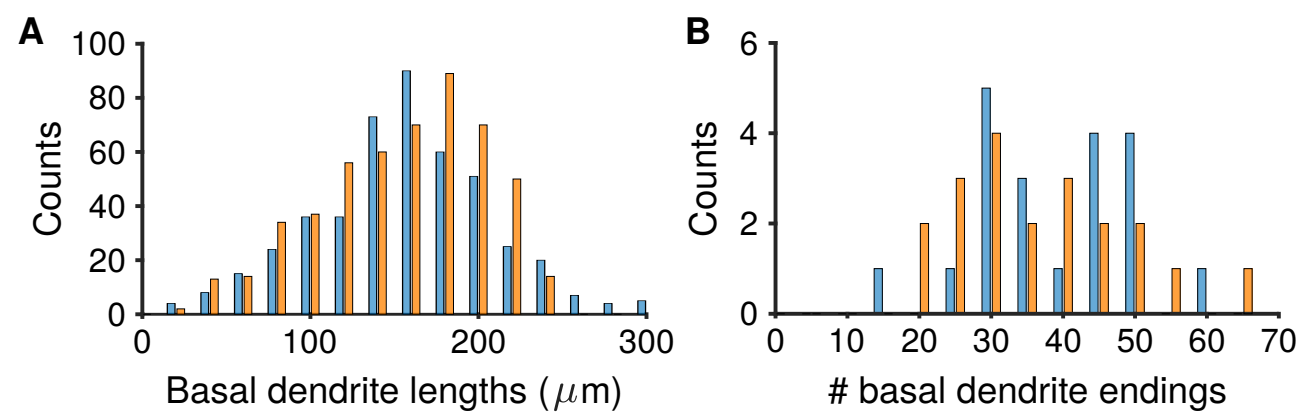

Supplementary Figure 7: A: Histogram of total basal dendrite length per cell from measured [60] and simulated data. B: Histogram of total number of basal dendrite endings 


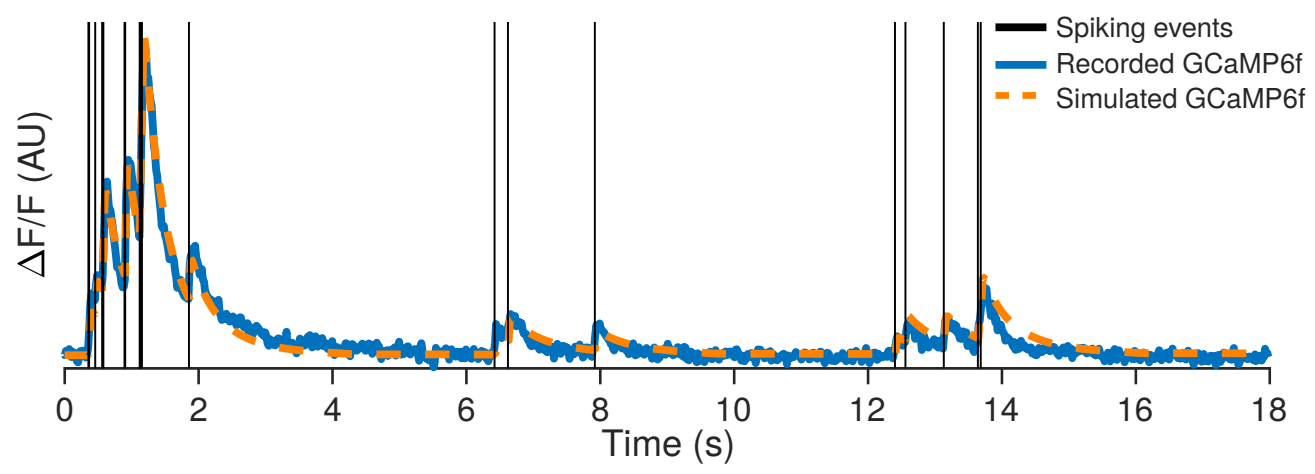

Supplementary Figure 8: Simultaneously recorded spikes and two-photon fluorescence timecourse [114] along with the estimated fluorescence timecourse using a forward model of calcium response.

(a)

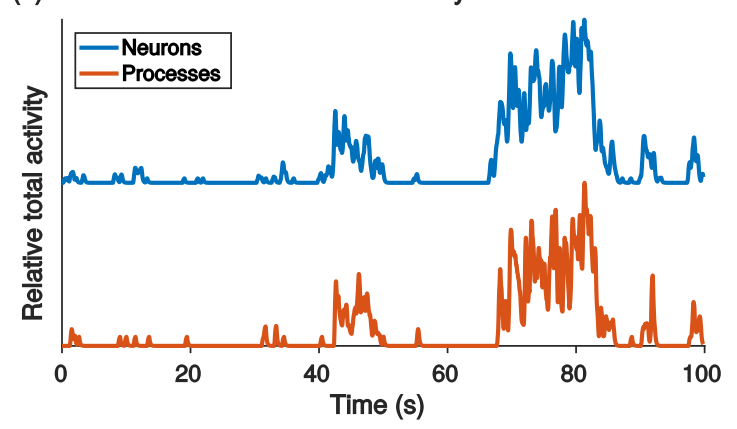

(b) Network traces

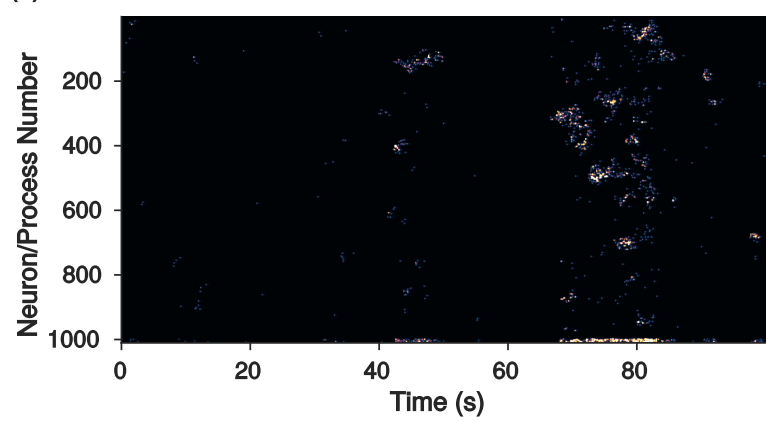

Supplementary Figure 9: Example of a Hawkes point-process. (a) The total activity of the network is correlated with the total activity in the background processes. (b) The Hawkes process gives the network- and single neuron- bursting statistics common in many neural activity recordings.
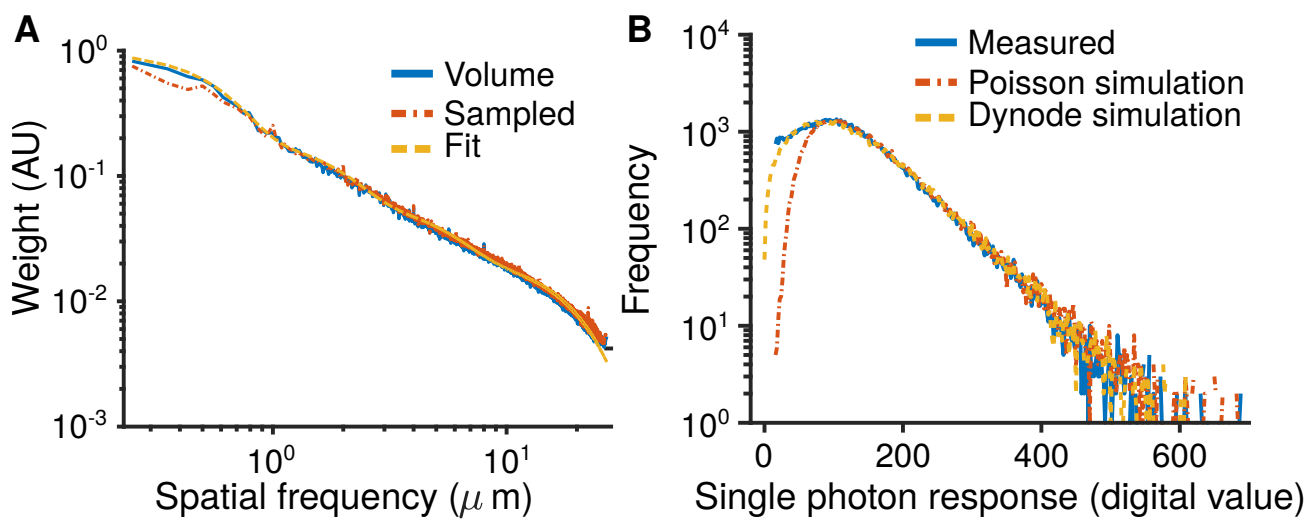

Supplementary Figure 10: A: Spatial frequency weights of an EM volume of mouse visual cortex, the fit to a Gaussian mixture model (GMM), and weights from a 3D Gaussian process sampling using the GMM. B: Measured PMT single photon response digital count distribution as compared to a Poisson amplification model or a dynode amplification model. 

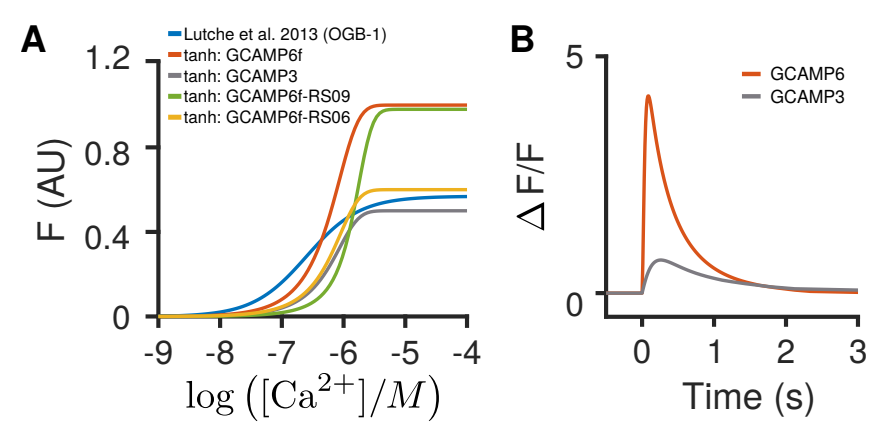

Supplementary Figure 11: A: Example hill plots that can be used in the fluorescence simulation (relating calcium concentration to $\Delta \mathrm{F} / \mathrm{F}$ ). The height and slope dictate the saturation and decay behavior. These curves were to the indicators in [71], using the parameters supplied therein. B: Example $\Delta \mathrm{F} / \mathrm{F}$ behavior for GcAMP6f and GcAMP6s in response to a ten-spike burst.

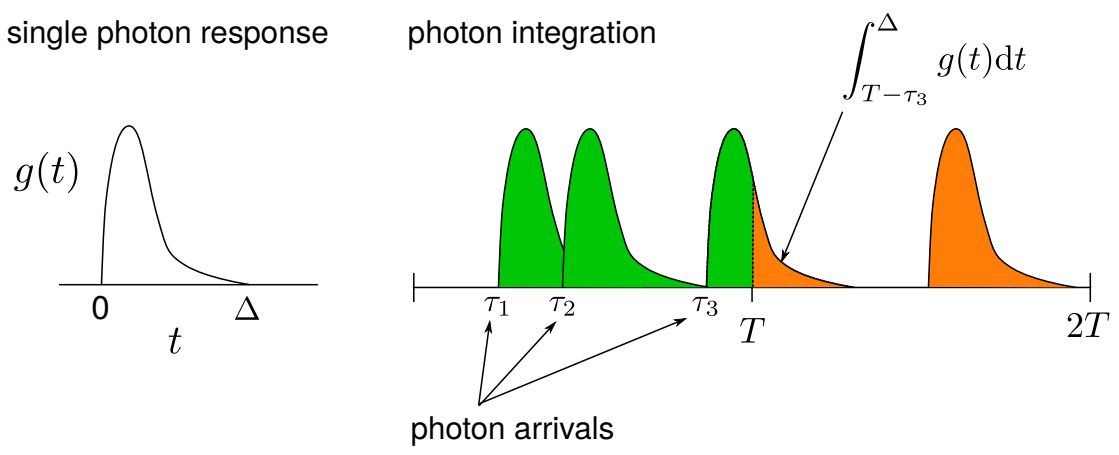

Supplementary Figure 12: Bleed-through of photon responses during TPM electronic analog-todigital conversion. Left: A single photon causes a response in the electronics that persists over a time frame $\Delta$. Right: any photons that arrive within $\Delta$ of the end of the sampling period cutoff (every $T$ seconds) have responses that are partially integrated into the current sample (green) and partially integrated into the next sample (orange). 
Single noiseless frame

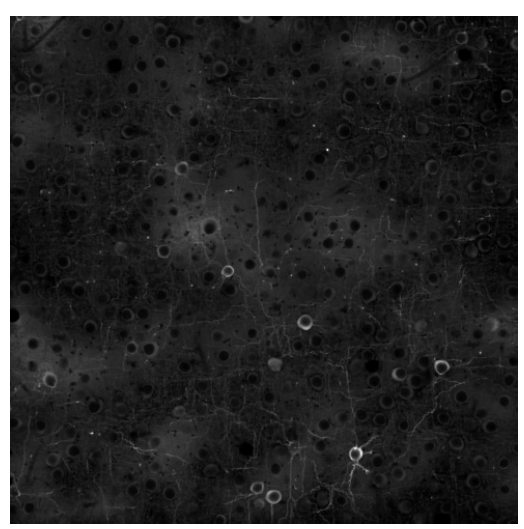

Ten-frame noiseless average

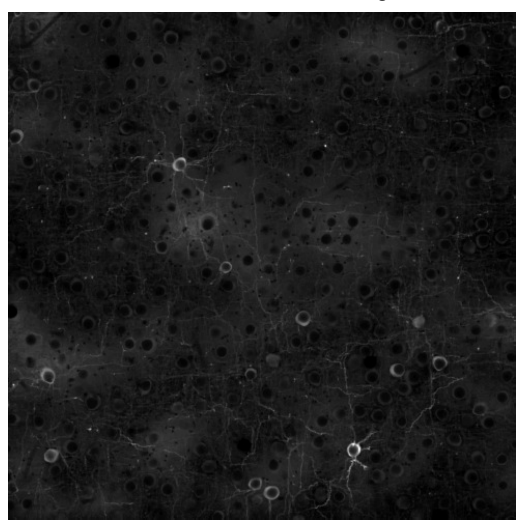

Single frame

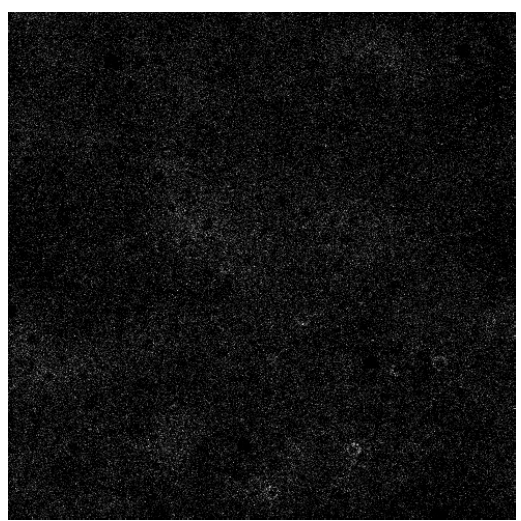

Ten-frame average

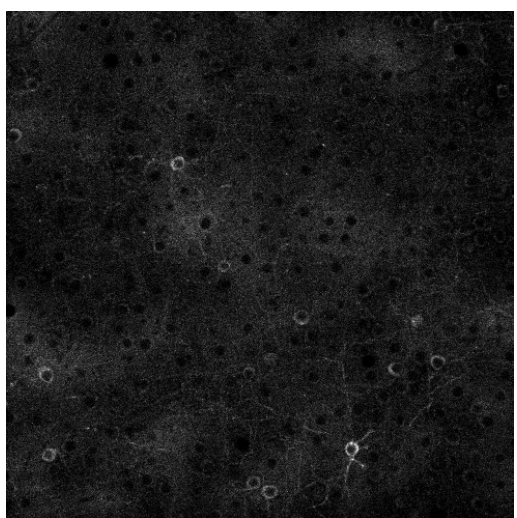

Single frame at $1 / 4$ power

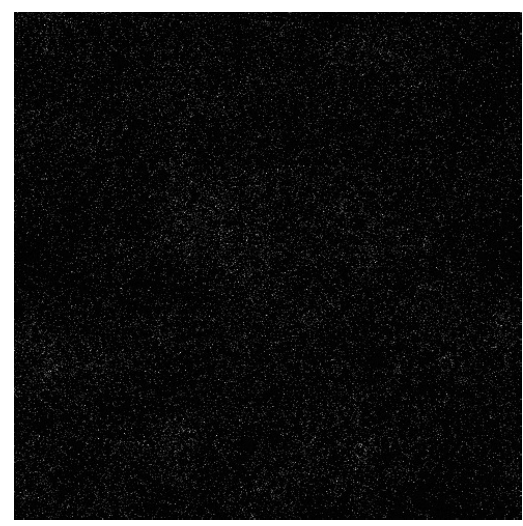

Ten-frame average at $1 / 4$ power

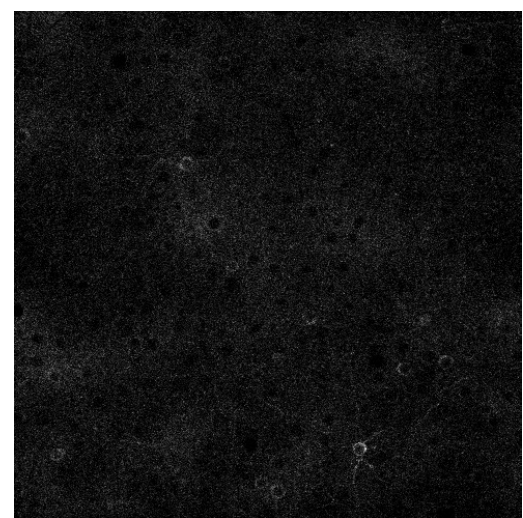

Supplementary Figure 13: Example frames showing the effect of reducing the power of the scanning PSF.
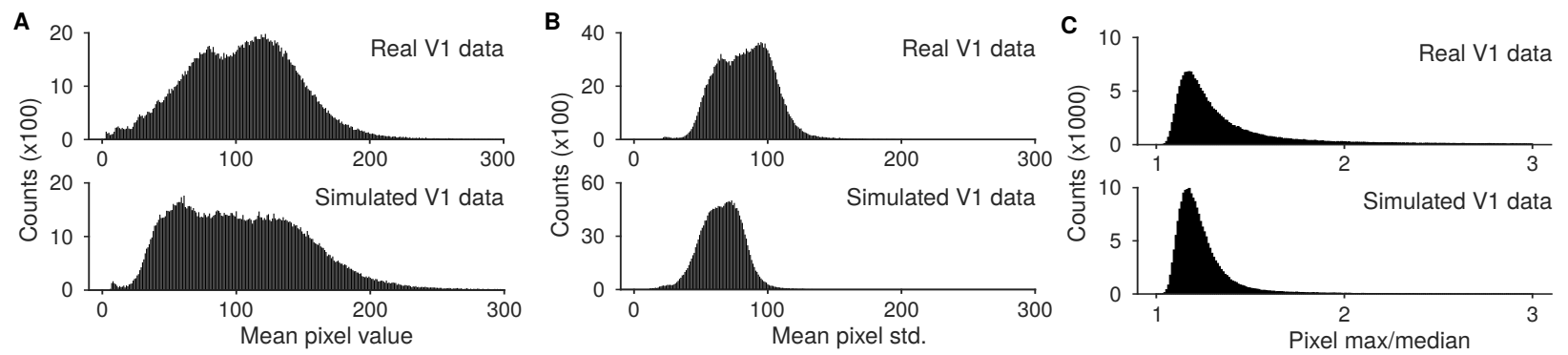

Supplementary Figure 14: A: Histogram of values in the mean image over 20000 frames for both real and simulated V1 data. B: Histogram of standard deviations across the FOV over 2000 frames for both real and simulated V1 data. C: Histogram of the ratio of the maximum value to the median value (approximate estimte of activity) across all pixels in the FOV, calculated over 20000 frames. 


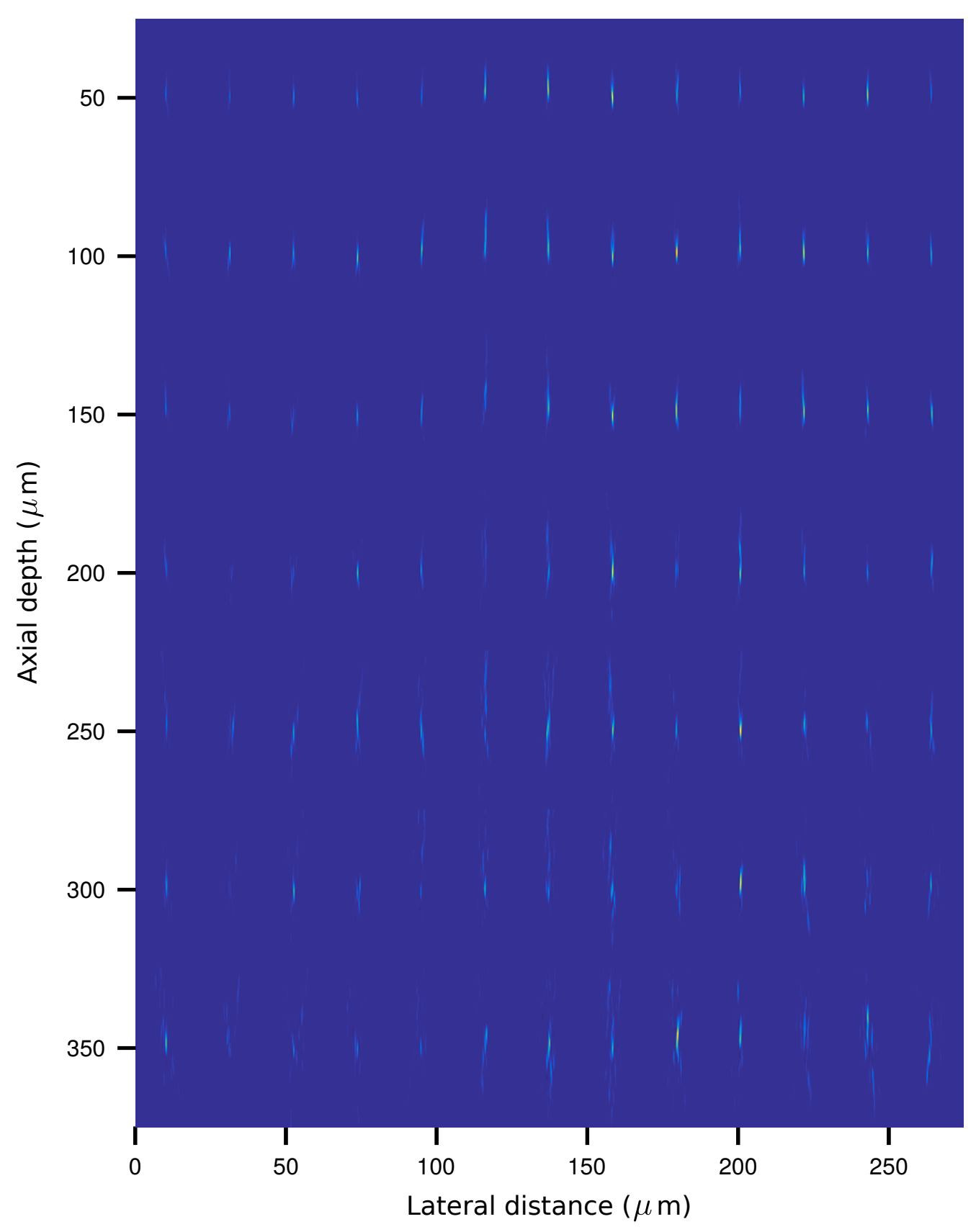

Supplementary Figure 15: Simulated propagation of a point-spread function through layers of diffusing tissue using NAOMi. Simulated PSFs equally spaced across two $\approx 2000 \mu \mathrm{m}$ cross sections of tissue, sampled at 

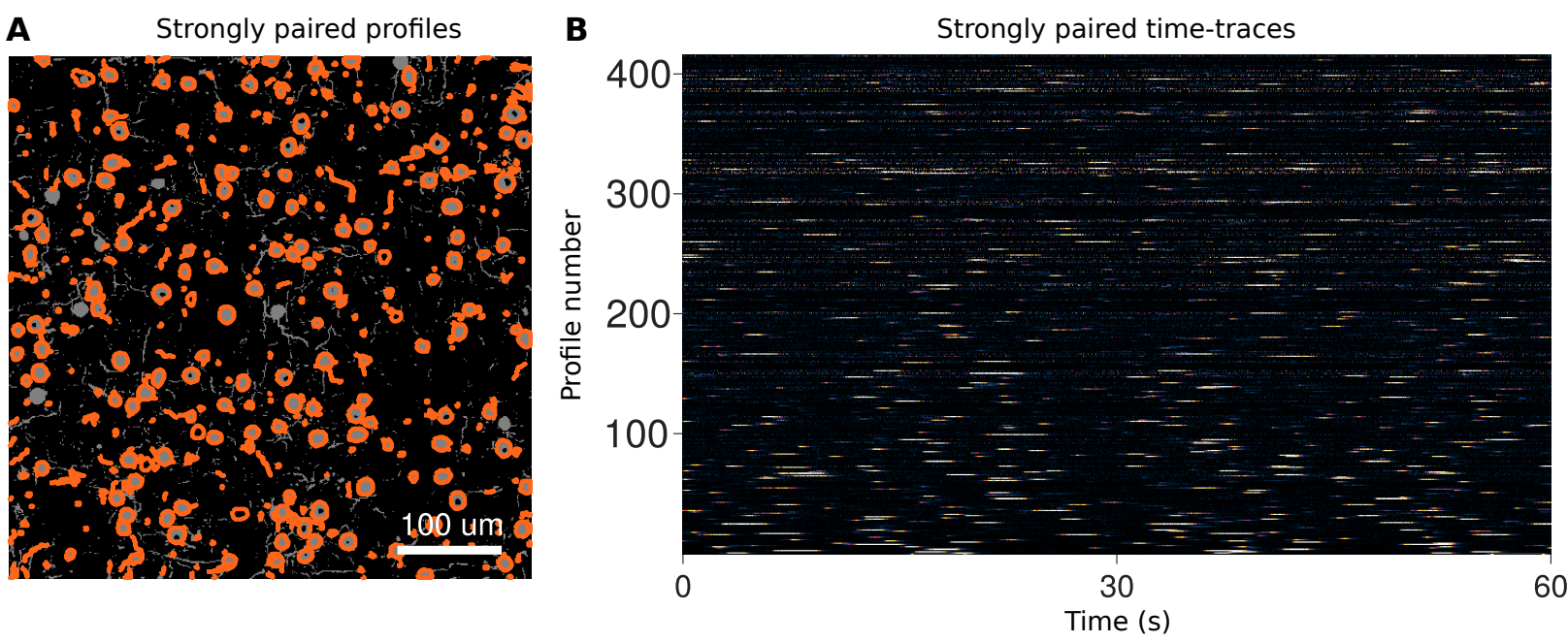

Supplementary Figure 16: Results of using the ideal spatial components to de-mix the simulated TPM video with frame-by-frame least-squares.

A

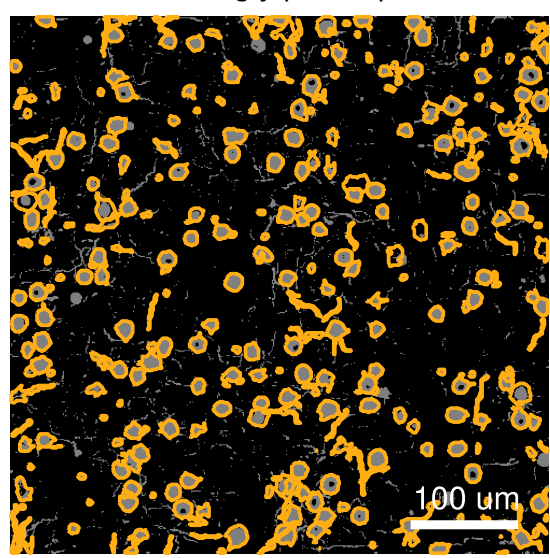

B

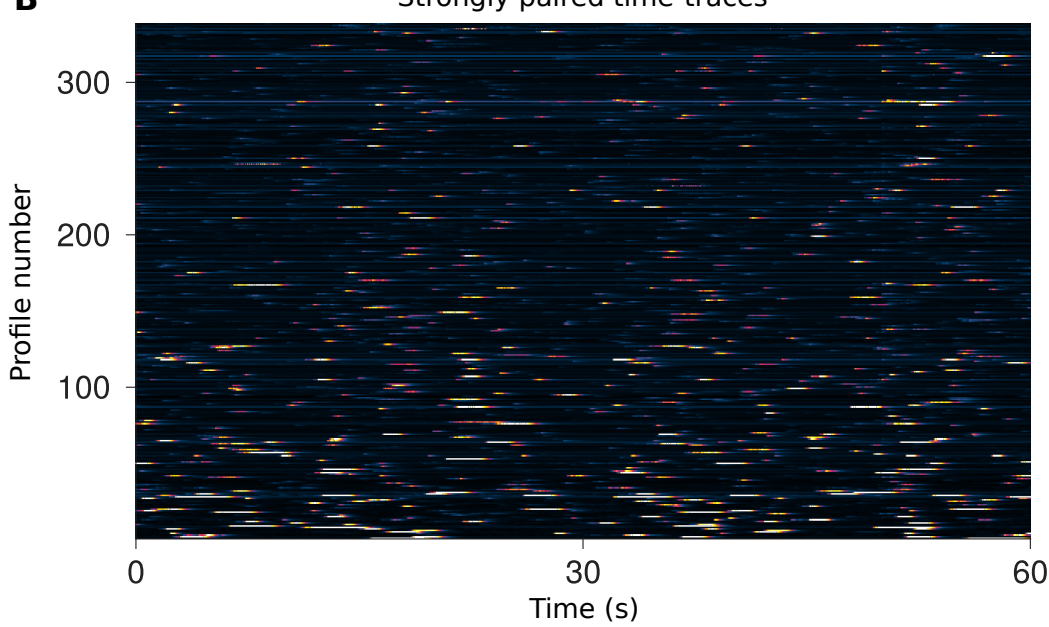

Supplementary Figure 17: Results of using CNMF to analyze the simulated TPM video. 

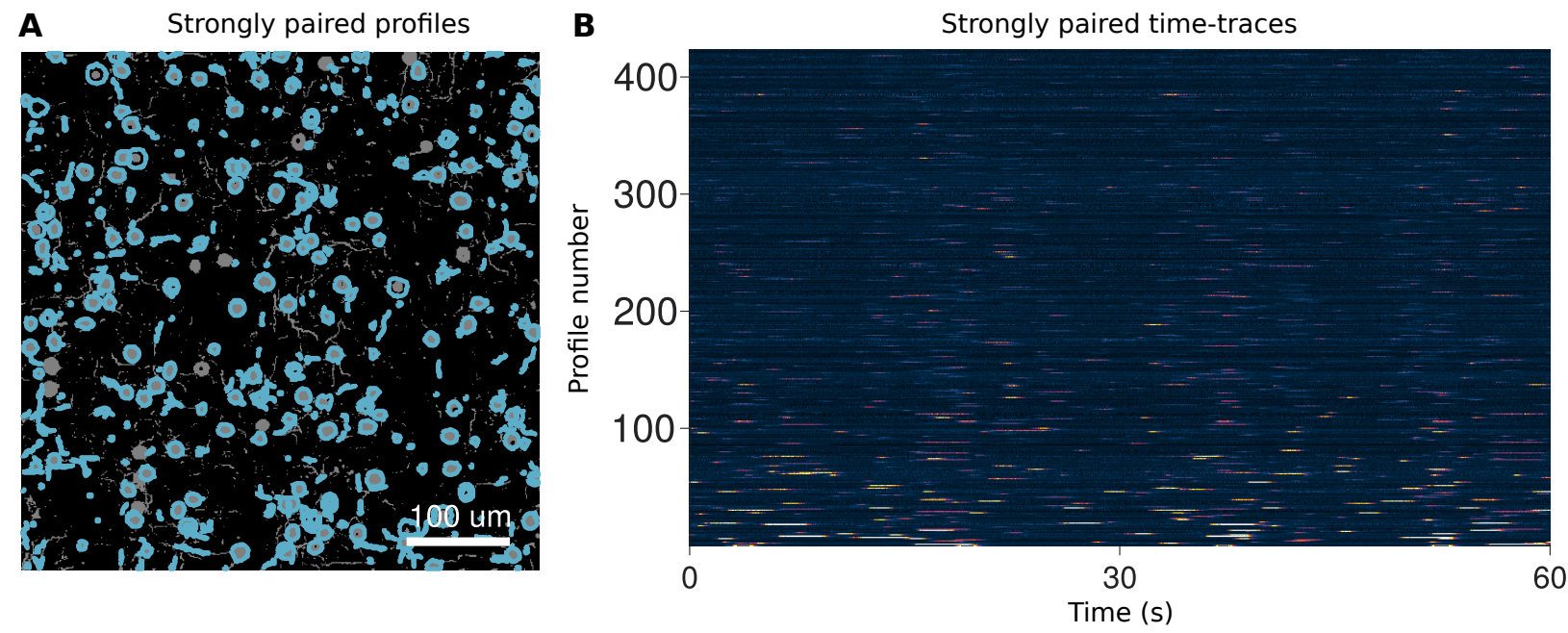

Supplementary Figure 18: Results of using Suite2p to analyze the simulated TPM video.

A

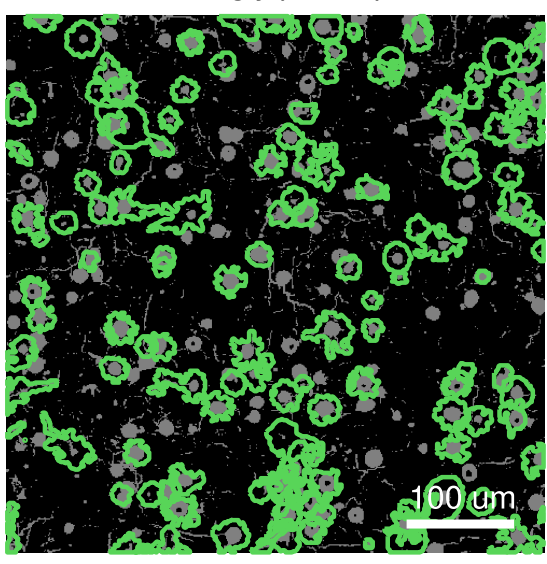

B

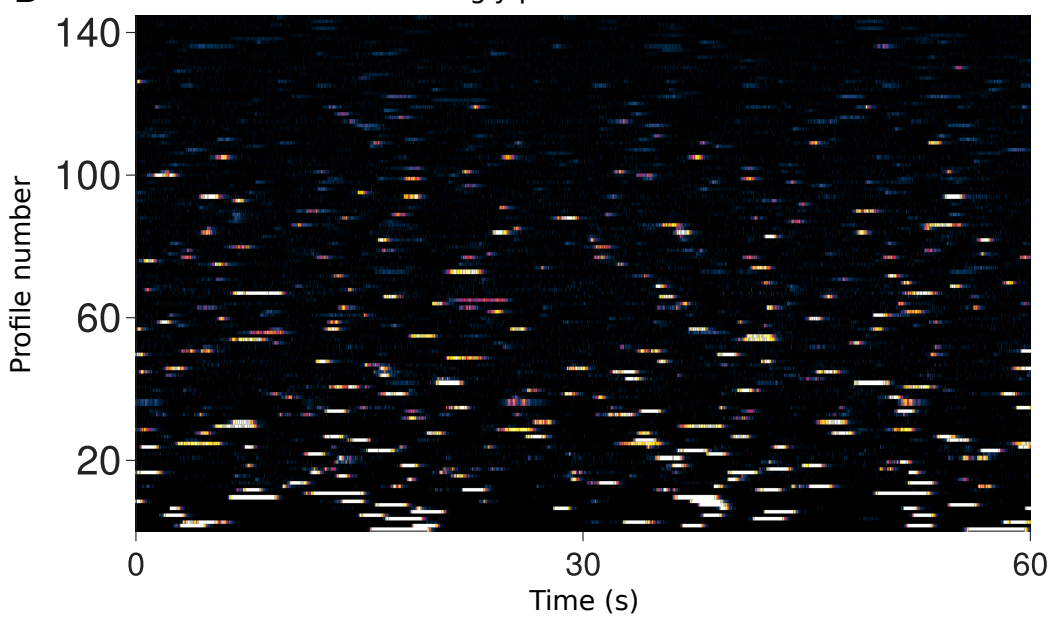

Supplementary Figure 19: Results of using the PCA/ICA to analyze the simulated TPM video. 


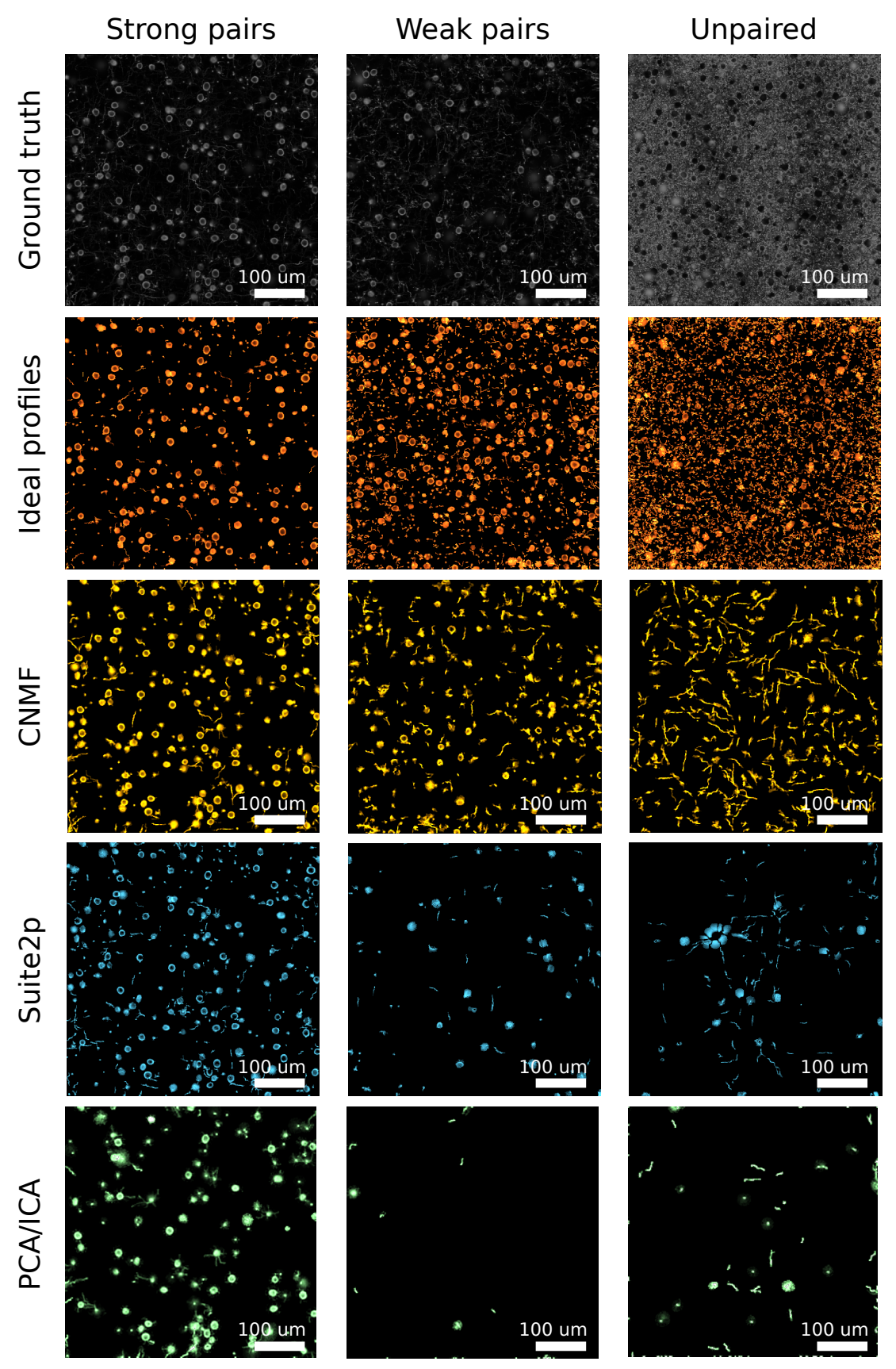

Supplementary Figure 20: Profile shapes for strongly paired profiles ( $\rho>0.5$; left column), weakly paired profiles $(0.1<\rho<0.5$; middle column) and unpaired profiles ( $\rho<0.1$; right column). Most cells (top row), were unpaired. Paired cells tended to be found via their somatic signal. CNMF (second row) tended to find the most profiles and matched the most cells. CNMF, however, also found the most false-positives, which tended to be dendritic shapes. Suite2p (third row) found both fewer cells and fewer false positives. PCA/ICA had the lowest number of found cells but still had a significant number of false positives. 


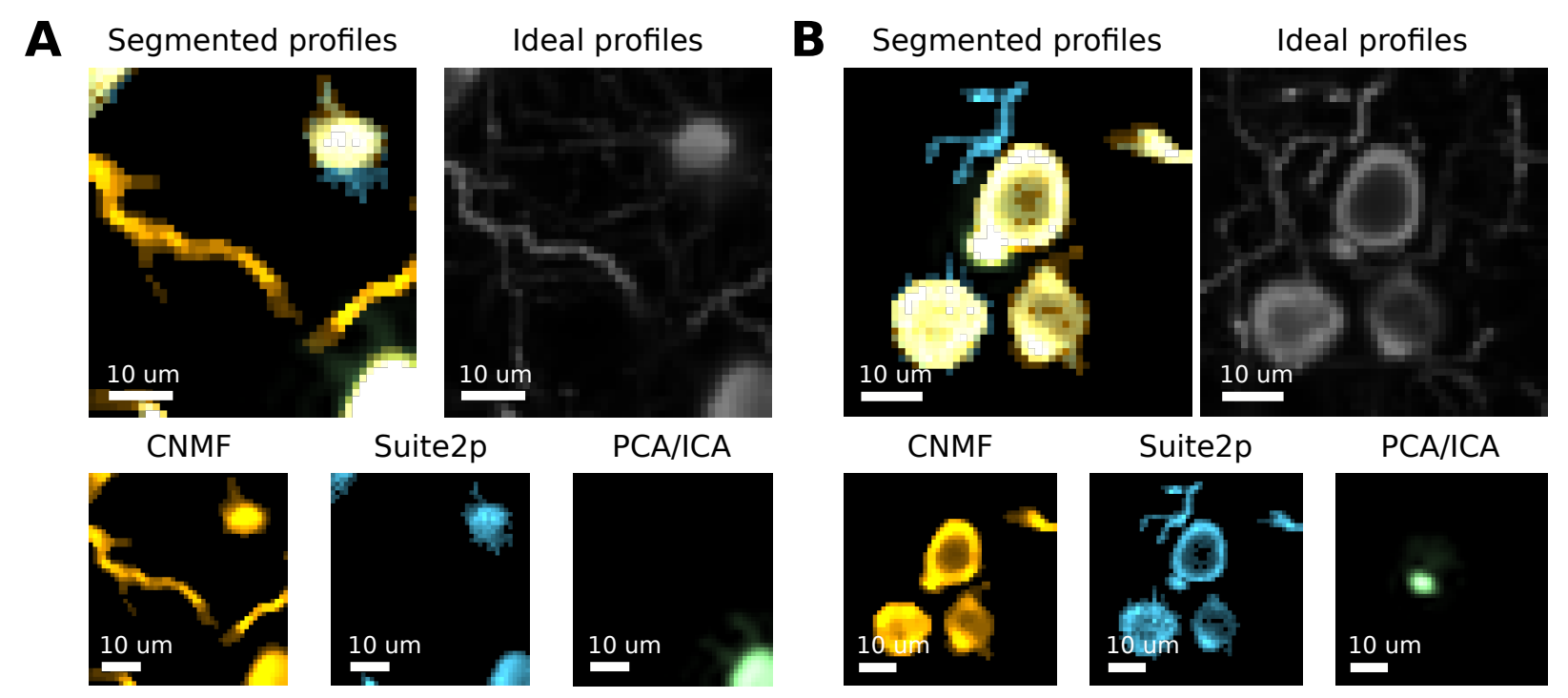

Supplementary Figure 21: Examples of finer features in the segmented profiles. Amongst the profiles found by the three different methods (CNMF in red, Suite2p in blue and PCA/ICA in green) somas were often found by multiple methods while finer features, such as dendrites were often found by only one algorithm. A: A segment containing somas found by both CNMF and Suite2p (one also found using PCA/ICA) and a dendrite found only with CNMF. B: A segment containing somas found by both CNMF and Suite2p (one also found using PCA/ICA) and a dendrite found only with Suite2p.
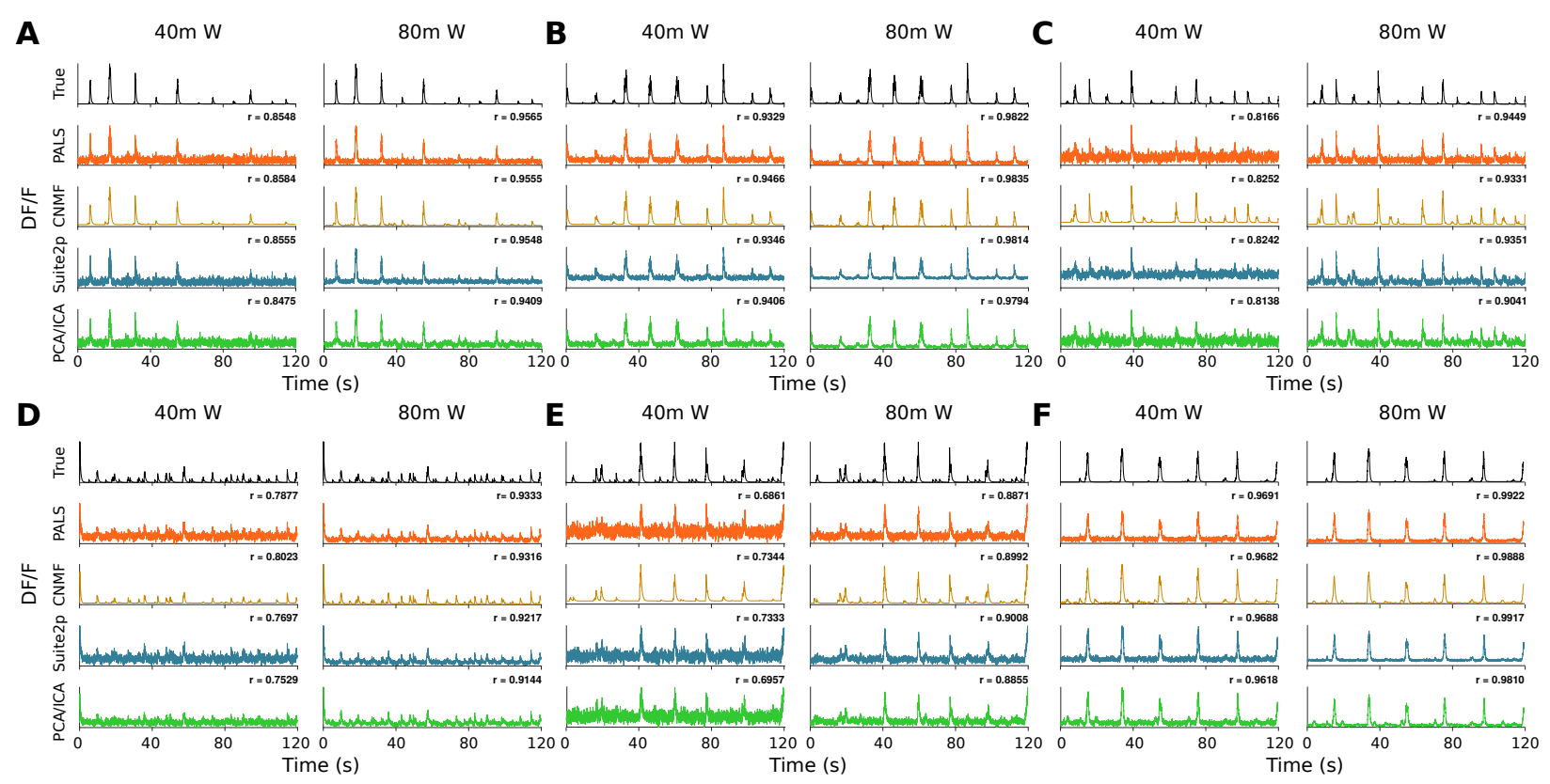

Supplementary Figure 22: Examples of strongly paired time-traces in simulations using 0.6-NA Gaussian beams $40 \mathrm{~m} \mathrm{~W}$ and $80 \mathrm{~m}$ W power. 

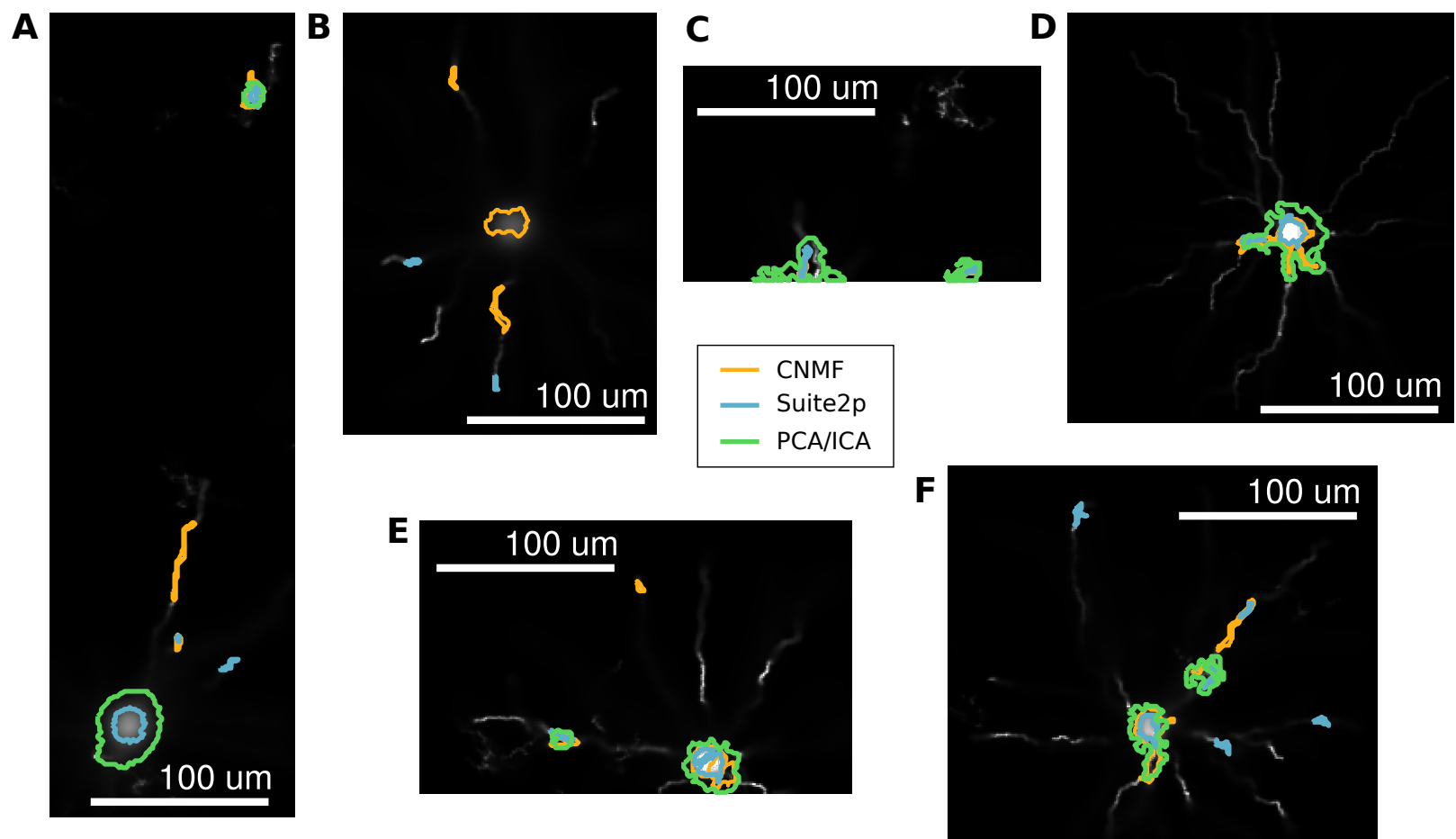

Supplementary Figure 23: Examples of cells that were found with multiplicity by one or more algorithms. A: Such cells can have profiles that are hundreds of $\mu \mathrm{m}$ away. B. More than two profiles can represent the same cell and often capture dendritic portions away from the soma. C: Such duplicity can happen when the F.O.V. cuts off a portion of the cell. D: Some examples were observed where the dendrite profile and soma profile were very close, where the profiles should have been merged. E: An example where two profiles represent the same soma and overlap. F: All three algorithms are susceptible to the multiplicity effect, proportionally to the number of profiles found. 
$40 \mathrm{~m} \mathrm{~W}$
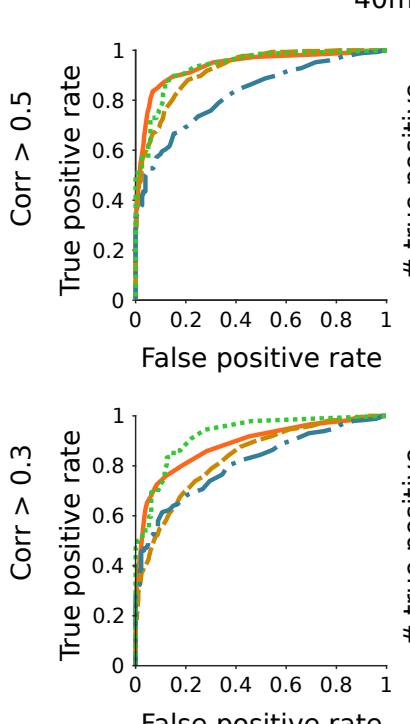

False positive rate

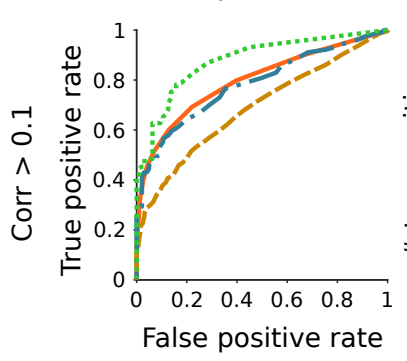

$\times 100$
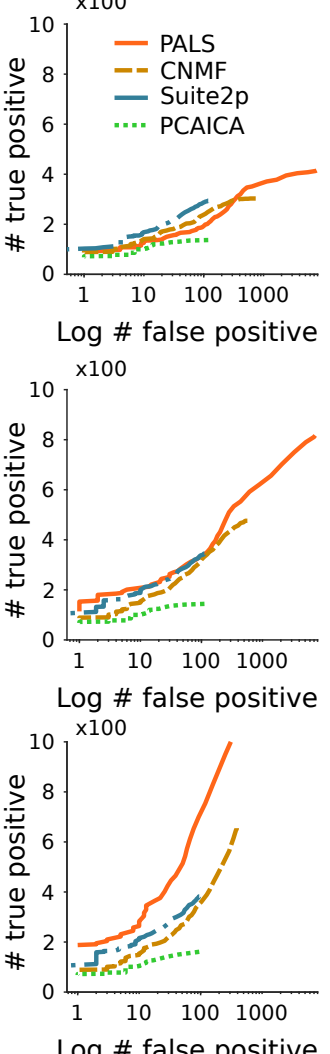

$80 \mathrm{~m} \mathrm{~W}$
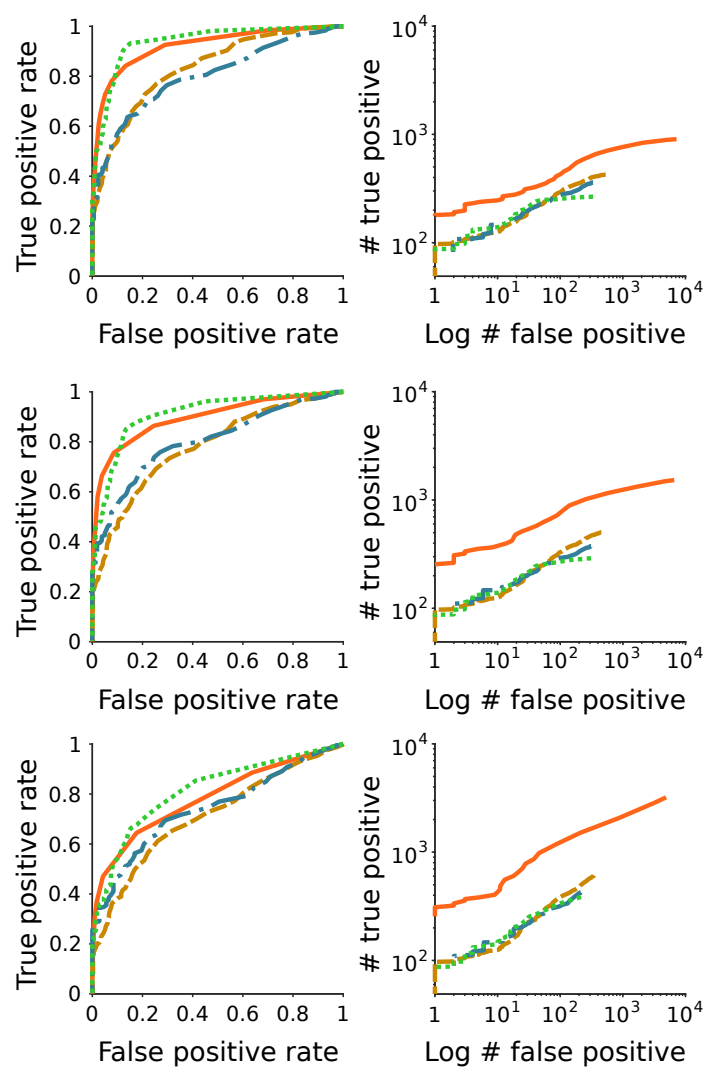

Supplementary Figure 24: Examples ROC curves at different power levels (40 $\mathrm{mW}$ on the left and $80 \mathrm{~mW}$ on the right) and with different correlation cutoffs for determining true vs. artifact sources (0.1, 0.3, and 0.5 in the three rows). Each set of curves show on the left the rates of true vs. false, as a fraction of the total number of true and artifact cells found by each algorithm, and on the right the total number of true and artifact sources. 
A
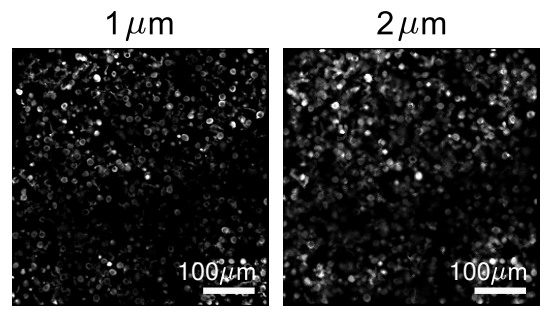

B

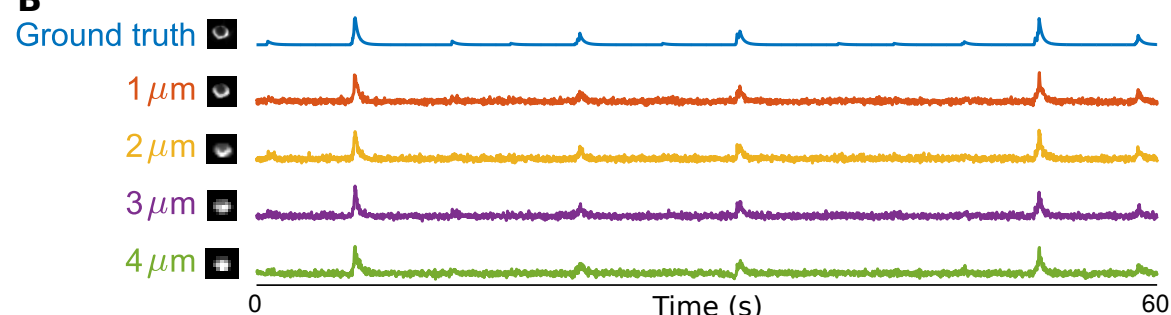

$4 \mu \mathrm{m}$

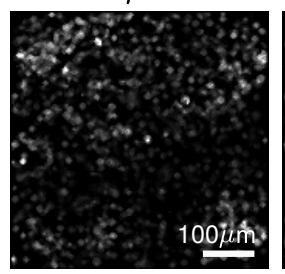

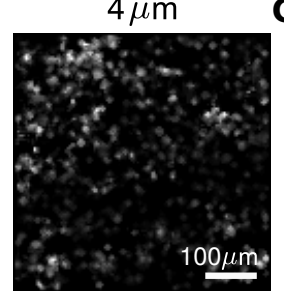

$0 \mu \mathrm{m}$

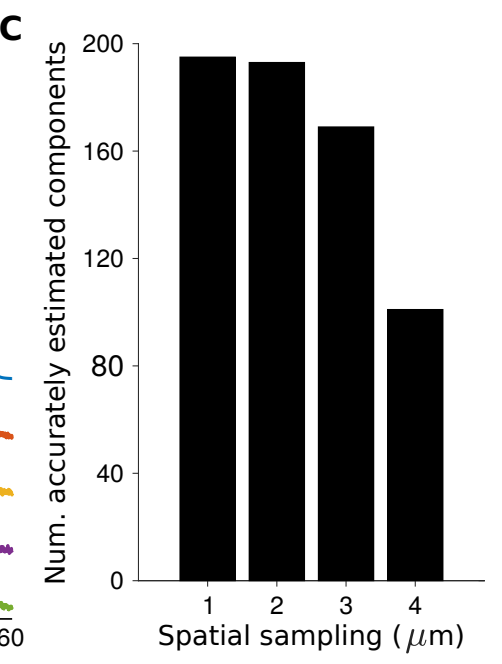

Supplementary Figure 25: Analysis of signal loss as a function of subsampling interval. A: Mean images calculated from imaging movies of the same volume and activity, taken at $1,2,3$, and $4 \mu \mathrm{m}$ intervals. B: Example cell spatial profile and time traces at all four image subsampling resolutions, as compared to the ground truth used to generate the data. C: Comparison of the number of cells confidently found (correlation $>0.5$ ) at all four resolutions indicate a steep fall-off after $3 \mu \mathrm{m}$ subsampling.
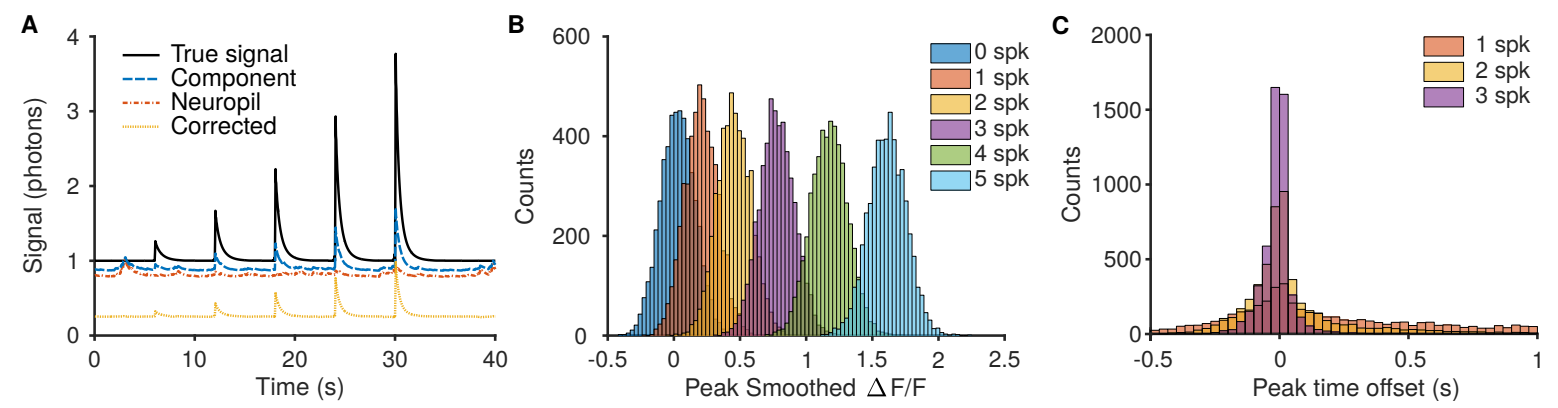

Supplementary Figure 26: Comparison of resulting $\Delta \mathrm{F} / \mathrm{F}$ distinguishability for bursts of different spike numbers. A: Simulations were run by generating videos with preset spiking activity that ramped up the number of spikes in each burst. Much of the error in recovered component timetraces can be attributed to insufficiently removed neuropil, and using algorithmic techniques such as in Suite2P [18] can increase the accuracy of the time traces. B: The recovered $\Delta \mathrm{F} / \mathrm{F}$ for larger bursts was more reliable and more separable between the different burst strengths. For example there is less overlap between the peak $\Delta \mathrm{F} / \mathrm{F}$ for 5 - and 4-spike bursts then for 1- and 2-spike bursts. $\mathrm{C}$ : The peak $\Delta \mathrm{F} / \mathrm{F}$ offset time is also more reliable for bursts with more spikes. 

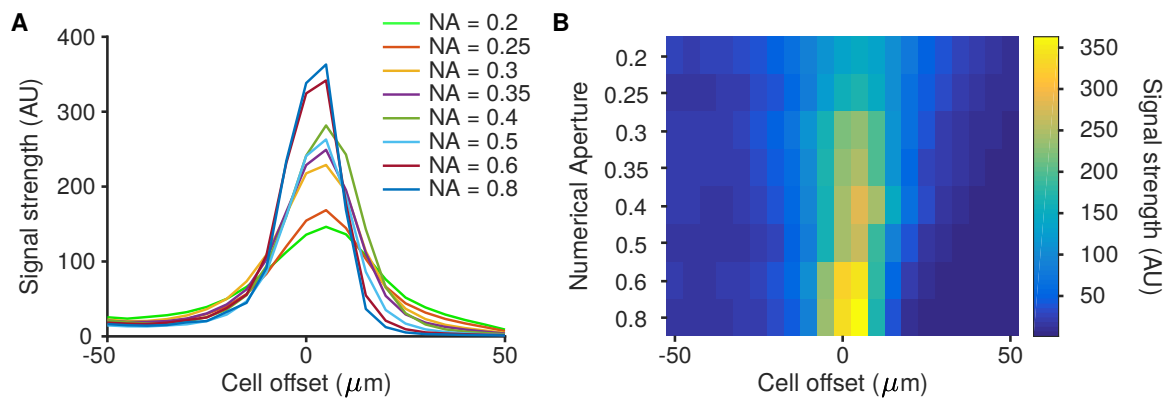

Supplementary Figure 27: Analysis of signal strength as a function of numerical aperture (NA). A: Signal strength drops off as a function of the displacement of the cell from the peak PSF excitation. B: Same data plotted as an image to better compare spread and peak intensity of the various curves. 